$\therefore \quad \begin{array}{r}1403 \\ 3803\end{array}$

NASA Contractor Report 4579

\title{
Long-Wavelength Asymptotics of Unstable Crossflow Modes, Including the Effect of Surface Curvature
}

Meelan Choudhari

(NASA-CR-4579) LONG-WAVELENGTH ASYMPTOTICS OF UNSTABLE CROSSFLOW MODES, INCLUDING THE EFFECT OF SURFACE CURVATURE (High Technology (OTP.) $43 \mathrm{p}$
N94-29460

Unclas

H1/34 0003803 
๑. . 


\section{Long-Wavelength Asymptotics of Unstable Crossflow Modes, Including the Effect of Surface Curvature}

Meelan Choudhari

High Technology Corporation • Hampton, Virginia 



\title{
Long-wavelength asymptotics of unstable crossflow modes, including the effect of surface curvature
}

\author{
Meelan Choudhari \\ High Technology Corporation \\ Hampton, VA 23666.
}

\begin{abstract}
Stationary vortex instabilities with wavelengths significantly larger than the thickness of the underlying three-dimensional boundary layer are studied with asymptotic methods. The long-wavelength Rayleigh modes are locally neutral and are aligned with the direction of the local inviscid streamline. For a spanwise wave number $\beta<<1$, the spatial growth rate of these vortices is $O\left(\beta^{3 / 2}\right)$. When $\beta$ becomes $O\left(R^{-1 / 7}\right)$, the viscous correction associated with a thin sublayer near the surface modifies the inviscid growth rate to the leading order. As $\beta$ is further decreased through this regime, viscous effects assume greater significance and dominate the growth-rate behaviour. The spatial growth rate becomes comparable to the real part of the wave number when $\beta=O\left(R^{-1 / 4}\right)$. At this stage, the disturbance structure becomes fully viscous-inviscid interactive and is described by the triple-deck theory. For even smaller values of $\beta$, the vortex modes become nearly neutral again and align themselves with the direction of the wall-shear stress. Thus, the study explains the progression of the crossflow-vortex structure from the inflectional upper branch mode to nearly neutral long-wavelength modes that are aligned with the wall-shear direction.
\end{abstract}




\section{INTRODUCTION}

Boundary-layer transition on a three-dimensional wing is often initiated by the so-called crossflow instability that owes its origin to the inflectional nature of the boundary-layer profile along the crossflow direction (i.e., the direction that is orthogonal to the local inviscid streamline). The crossflow instability was first observed by Gregory and Walker (Gregory, Stuart, and Walker, 1955) using napthalene flow visualization, which indicated the vortex instability to be stationary relative to the airfoil surface. A theoretical explanation for this instability was given by Stuart in the same paper. He showed that the above disturbance modes are governed by Rayleigh's equation, which is based on the boundary-layer profile along the direction of the disturbance wave-number vector. Stuart also went on to demonstrate that the Rayleigh problem permits unstable eigenmodes for each transverse wave number (i.e., wave number in the spanwise or the azimuthal direction, depending on the body geometry) which is smaller than some critical value. The critical (i.e., neutral) wave number was shown by Stuart to correspond to that normal mode for which the inflection point of the directional mean profile coincides with the wall-normal location where the directional speed is equal to zero.

The experiments of Gregory et al.(1955) established the rotating-disk boundary layer as a laboratory prototype for studying the crossflow-vortex instability. Viscous stability calculations for this flow were carried out by Malik (1986), who demonstrated the importance of retaining the Coriolis terms in the relevant (quasi-parallel) stability equations and also discovered the longwavelength (or lower) branch of the neutral stability curve in the wave-number Reynolds-number plane. The asymptotic structure of these lower branch modes was simultaneously explained by Hall (1986). He showed that the lower branch vortices are aligned with the direction of the mean wall shear stress and, furthermore, are significantly influenced by the viscous and Coriolis forces. Hall also calculated the leading viscous correction to the inviscid (i.e., Rayleigh mode) neutral solution (generally referred to as the upper branch mode) which was investigated earlier by Stuart (Gregory, Stuart, and Walker, 1955).

If the Rayleigh mode calculation for the rotating-disk boundary layer is continued from the upper branch wave number toward smaller values of the azimuthal wave number, then the radial amplification rate increases initially, reaches its maximum at some finite wave number and, finally, falls back to zero again as the azimuthal wave number tends to zero (Choudhari 
and Streett, unpublished results, 1990). However, somewhat surprisingly, the limiting form of the long-wavelength modes corresponds to vortices that are aligned with the local free-stream direction, against the viscous Coriolis-balanced vortices of Hall (1986), which are aligned with the direction of the mean wall shear stress. A similar observation was recently made by Blackaby and Choudhari (1993) in the context of the Falkner-Skan-Cooke (FSC) family of boundary layers, in which the Coriolis forces are absent altogether. Because Coriolis forces are also absent from the boundary-layer flows encountered in typical aeronautical applications, an investigation of the properties of the long-wavelength Rayleigh modes is deemed important; this investigation is the purpose of the present article.

In section 2, we show that the long-wavelength inviscid vortices are neutral to the leading order and that their relatively small amplification rate is determined by the local sweep angle and the momentum integral associated with the jetlike crossflow profile. The latter dependence is characterized analytically, and the similarity of the result to that obtained earlier by Drazin and Howard (1962) in the case of unbounded, two-dimensional jets and wakes is pointed out. The stabilizing effects of convex surface curvature on the long-wavelength crossflow instability are considered in section 3, wherein we elucidate the asymptotic behaviour of the relevant neutral branch found by Blackaby and Choudhari (1993). Perhaps the most significant difference between the case of free-shear flows examined by Drazin and Howard (1962) and the boundarylayer case examined herein is related to the earlier onset of viscous effects in the present problem. The role of viscosity in modifying inviscid growth-rate behaviour as the transverse disturbance wave number $\beta$ decreases through the successive regimes of $\beta=O\left(R^{-1 / 7}\right), \beta=O\left(R^{-1 / 4}\right)$, and $\beta=O\left(R^{-3 / 7}\right)$ is discussed in sections $4.1,4.2$, and 4.3 , respectively. Concluding remarks based on this study are presented in section 5 .

\section{ANALYSIS}

For a three-dimensional mean boundary layer, the profiles along a pair of suitably chosen Cartesian axes $x^{*}$ and $z^{*}$ along the surface are assumed to be given by $\mathcal{V}^{*} \cos \theta_{s w} \bar{u}(Y ; x, z)$ and $\mathcal{V}^{*} \sin \theta_{s w} \bar{w}(Y ; x, z)$, respectively. (See figure 1.) Here, $x=x^{*} / \ell^{*}$ and $z=z^{*} / \ell^{*}$ are the nondimensional coordinates based on the length scale $\ell^{*}$ over which the boundary-layer flow evolves; $Y=y^{*} / L^{*}$ is the wall-normal coordinate scaled by a typical thickness $L^{*}$ of the boundary layer; 
and $\theta_{s w}$ is the local sweep angle, so that $\bar{u}(Y)$ and $\bar{w}(Y)$ represent the normalized profiles at station $(x, z)$ (i.e., $\lim _{Y \rightarrow \infty} \vec{u}(Y)=\lim _{Y \rightarrow \infty} \bar{w}(Y)=1$ ). Without any loss of generality, we will assume that $-\pi / 2<\theta_{s w}<\pi / 2, \cos \theta_{s w}=O\left(\sin \theta_{s w}\right)$ (so that the local free stream is not aligned with either the $x$ or the $z$ axis) and that $\bar{u} \neq \bar{w}$ for all $Y$ (so that the base flow is genuinely three dimensional). In the case of swept-wing boundary layers in low-speed flows, it is usually true that the velocity profile along the direction of the inviscid streamline (i.e., $\cos ^{2} \theta_{s w} \bar{u}+\sin ^{2} \theta_{s w} \bar{w}$ ) has a monotonic behavior in $Y$. Also, the crossflow profile $\left(\equiv \cos \theta_{s w} \sin \theta_{s w}(\bar{u}-\bar{w})\right)$ reaches its maximum magnitude within the boundary layer and exhibits a single inflection point between the location of the above maximum and the edge of the boundary layer where the crossflow magnitude tends to zero. In order any possible complications associated with more intricate boundary-layer profiles, we will restrict our attention to profiles that satisfy the above properties.

As stated in the introduction, the objective of our analysis is to trace the evolution of the crossflow-vortex characteristics as the wavelength of the vortex is increased gradually, starting from its value for the inflectional, upper-branch mode. It is shown in sections 2 and 4.1 below that in the first two asymptotic subregimes encountered during this process, the disturbance structure involves an asymptotic subregion near the edge of the mean boundary layer, where the crossflow magnitude is asymptotically small. In order to simplify the analysis, we assume that the mean-flow vorticity decays at an exponential rate as $Y \rightarrow \infty$. That is,

$$
\bar{u} \sim 1-C_{\bar{u}} \exp \left[-\kappa_{u}\left(Y-Y_{\delta}\right)\right]
$$

and

$$
\bar{w} \sim 1-C_{\bar{w}} \exp \left[-\kappa_{w}\left(Y-Y_{\delta}\right)\right]
$$

where $C_{\bar{u}}, C_{\bar{w}}, \kappa_{u}, \kappa_{w}$, and $Y_{\delta}$ are known constants. In order to ensure that the variations in both $\bar{u}$ and $\bar{w}$ are significant in the abovementioned edge region (where $Y>>1$ ) we assume that $\kappa_{u}=\kappa_{w}=\kappa$. However, we shall demonstrate that the specific form of the decay in meanflow vorticity is unimportant in determining the leading-order growth rate of the crossflow eigenmodes. Hence, the results obtained herein are equally applicable to boundary-layer profiles that have, for instance, a faster-than-exponential decay of vorticity into the free stream (e.g., the self-semilar Falkner-Skan-Cooke boundary layers). To minimize the influence of any extraneous 
parameters, the curvature of the surface within the region of interest is also assumed to be negligible to begin with.

\subsection{Inviscid Treatment}

Now consider the linear, normal-mode disturbance with a spanwise wave number equal to $\beta^{*}=$ $\beta / L^{*}, \beta(>0)=O(1)$. As shown by Hall (1986), the dynamics of this disturbance is governed by the Rayleigh's equation up to $O\left(R^{-1 / 3}\right)$, where $R(>>1)$ is the Reynolds number based on the velocity $\mathcal{V}^{*}$ and length $L^{*}$. Thus, the perturbation quantities in the boundary-layer region expand as

$$
\left(\frac{u^{*}}{\mathcal{V}^{*}}, \frac{v^{*}}{\mathcal{V}^{*}}, \frac{w^{*}}{\mathcal{V}^{*}}, \frac{p^{*}}{\rho \mathcal{V}^{* 2}}\right)=\Delta[u(Y), v(Y), w(Y), p(Y)] \exp [i(\alpha X+\beta Z)]
$$

where the parameter $\Delta(<<1)$ characterizes the infinitesimal disturbance amplitude; $X=x^{*} / L^{*}$ and $z=z^{*} / L^{*}$ are the "fast" coordinates scaled by the boundary-layer thickness $L^{*}$; and $\alpha$ is the complex wave number along the $X$ direction, which is the eigenvalue to be determined during the course of the analysis. The profiles $[u(Y), v(Y), w(Y), p(Y)]$ represent the corresponding eigenfunctions that illustrate the variation of the respective disturbance quantities along the wall-normal direction. In particular, $v(Y)$ is given by the solution to the Rayleigh's equation

$$
\mathcal{L}_{R} v \equiv\left(\alpha \cos \theta_{s w} \bar{u}+\beta \sin \theta_{s w} \bar{w}\right)\left[v^{\prime \prime}-\left(\alpha^{2}+\beta^{2}\right) v\right]-\left(\alpha \cos \theta_{s w} \bar{u}^{\prime \prime}+\beta \sin \theta_{s w} \bar{w}^{\prime \prime}\right) v=0
$$

which also satisfies the homogeneous boundary conditions

$$
v(0)=0 \text { and } \quad v(Y) \rightarrow 0 \text { as } Y \rightarrow \infty
$$

The primes in (3a) denote derivatives with respect to the wall-normal coordinate $Y$; unless specified otherwise, the same convention will be valid throughout this paper.

The objective of our long-wavelength analysis is to determine the asymptotic behaviour of the solution to the eigenvalue problem $(3 \mathrm{a}-\mathrm{c})$ when the transverse wave number $\beta$ tends to zero (i.e., $R^{-\tau}<<\beta<<1$ for any $\tau>0$ ). As mentioned in the introduction, numerical calculations suggest that $\alpha \sim-\beta \tan \theta_{s w}$ as $\beta \rightarrow 0$ because the vortices tend to align themselves with the local direction of the inviscid streamline. As a result, only two disparate length scales exist in the long-wavelength problem, namely the boundary-layer thickness $L^{*}$ and the disturbance 
wavelength $2 \pi / \beta^{*}$. Therefore, one would intuitively expect the disturbance structure to split into at least two distinct subregions along the surface-normal direction, namely those that correspond to $Y=O(1)$ and $Y_{u} \equiv \beta Y=O(1)$, respectively. However, the form of the asymptotic series relevant to either of these subregions cannot be predicted on an a priori basis because of the singular nature of the limit $\beta \rightarrow 0$.

The correct form of the asymptotic expansion can be determined with the benefit of some hindsight based on a knowledge of the properties of Rayleigh's equation, although in our derivation we were also guided by the closely related analysis of Papageorgiou and Smith (1989) on the long-wavelength stability of two-dimensional wake profiles. In view of this, the issue of justification for the form of asymptotic expansion used is postponed until later; the reader is asked to assume until then that the expansion for both the eigenvalue $\alpha$ and the eigenfunction $v(Y)$ across all the relevant subregions proceeds in integral powers of $\beta^{1 / 2}$ (at least up to the order required to determine the leading-order growth rate). Given this form of the expansion, we find that in addition to the two subregions already mentioned (i.e., $Y=O(1)$ and $Y_{u}=O(1)$ ) a third, intervening region that has the same thickness as that of the boundary layer and is located near the outer edge must be considered at a distance of $O(\log \beta)^{1 / 2}$ from the surface, where the magnitude of the crossflow velocity is $O\left(\beta^{1 / 2}\right)$.

We now begin the solution procedure by rewriting the Rayleigh's equation in a form that is suitable for long-wavelength analysis. Thus, (3a) is expressed in the form

$$
\mathcal{L}_{R} v \equiv\left(\mathcal{L}_{\mathcal{W}}+\beta^{2} \mathcal{M}\right) v=0
$$

The two operators $\mathcal{L}_{\mathcal{W}}$ and $\mathcal{M}$ are defined by

$$
\mathcal{L}_{\mathcal{W} v} \equiv\left[\mathcal{W}^{2}\left(\frac{v}{\mathcal{W}}\right)^{\prime}\right]^{\prime}
$$

and

$$
\mathcal{M} v \equiv-\left(1+\tan ^{2} \theta_{s w} \hat{\alpha}^{2}\right) \mathcal{W} v,
$$

wherein we have introduced new notation in the form of a scaled eigenvalue parameter

$$
\hat{\alpha} \equiv \alpha / \beta \tan \theta_{s w}
$$

and the normalized directional profile

$$
\mathcal{W}(Y) \equiv \hat{\alpha} \bar{u}(Y)+\bar{w}(Y)
$$


In anticipation of the result quoted earlier, $\hat{\alpha}$ expands in the form

$$
\hat{\alpha} \sim-1+\beta^{1 / 2} \alpha_{1}+\beta \alpha_{2}+\beta^{3 / 2} \alpha_{3}+\beta^{2} \alpha_{4}+\ldots \quad(\beta \rightarrow 0)
$$

Therefore, the operator norms for both $\mathcal{L}_{\mathcal{W}}$ and $\mathcal{M}$ continue to be of $O(1)$ in the main boundarylayer region during the above limiting process.

For $Y=O(1),(4 \mathrm{f})$ implies that

$$
\mathcal{W}(Y) \sim \mathcal{W}_{0}+\beta^{1 / 2} \alpha_{1} \bar{u}+\beta \alpha_{2} \bar{u}+\beta^{3 / 2} \alpha_{3} \bar{u}+\beta^{2} \alpha_{4} \bar{u}+\ldots \quad\left[\mathcal{W}_{0}(Y)=\bar{w}(Y)-\bar{u}(Y)\right]
$$

Accordingly, the eigenfunction $v(Y)$ can be expanded as

$$
v(Y)=\beta v_{0}(Y)+\beta^{3 / 2} v_{1}(Y)+\beta^{2} v_{2}(Y)+\beta^{5 / 2} v_{3}(Y)+\beta^{3} v_{4}(Y)+\ldots
$$

where the leading order perturbation has been taken to be $O(\beta)$ so that the magnitudes of other disturbance quantities $u, w$, and $p$ remain $O(1)$ even in the limit $\beta \rightarrow 0$. If we substitute the expansion (5b) into (4a) and use (4f) and (5a) with $\beta \rightarrow 0$, we find that $v_{n}, n=1-4$ satisfy

$$
\begin{gathered}
\mathcal{L}_{\mathcal{W}_{0}} v_{0}=0, \quad v_{0}(0)=0 ; \\
\mathcal{L}_{\mathcal{W}_{0}} v_{1}=-\alpha_{1} \mathcal{L}_{\bar{u}} v_{0}, \quad v_{1}(0)=0 ; \\
\mathcal{L}_{\mathcal{W}_{0}} v_{2}=-\alpha_{2} \mathcal{L}_{\bar{u}} v_{0}-\alpha_{1} \mathcal{L}_{\bar{u}} v_{1}, \quad v_{2}(0)=0 ; \\
\mathcal{L}_{\mathcal{W}_{0}} v_{3}=-\alpha_{3} \mathcal{L}_{\bar{u} v_{0}}-\alpha_{2} \mathcal{L}_{\bar{u}} v_{1}-\alpha_{1} \mathcal{L}_{\bar{u}} v_{2}, \quad v_{3}(0)=0 ;
\end{gathered}
$$

and

$$
\mathcal{L}_{\mathcal{W}_{0}} v_{4}=-\alpha_{4} \mathcal{L}_{\bar{u}} v_{0}-\alpha_{3} \mathcal{L}_{\bar{u}} v_{1}-\alpha_{2} \mathcal{L}_{\bar{u}} v_{2}-\alpha_{1} \mathcal{L}_{\bar{u}} v_{3}-\mathcal{M}_{0} v_{0}, \quad v_{4}(0)=0
$$

where

$$
\mathcal{L}_{\mathcal{W}_{0}} v \equiv\left[\mathcal{W}_{0}^{2}\left(\frac{v}{\mathcal{W}_{0}}\right)^{\prime}\right]^{\prime}, \quad \mathcal{L}_{\bar{u}} v \equiv\left[\bar{u}^{2}\left(\frac{v}{\bar{u}}\right)^{\prime}\right]^{\prime}, \quad \text { and } \quad \mathcal{M}_{0}=-\sec ^{2} \theta_{s w} \mathcal{W}_{0} . \quad(10 c, d, e)
$$

The behaviour of $v_{n}(Y), n=1-4$, when $Y \rightarrow \infty$ has not been specified at this stage, because it is determined by asymptotic matching with the solution from the adjacent outer region.

The general solution to the homogeneous, long-wavelength Rayleigh equation (6a) is given by

$$
v_{0}(Y)=C_{A} v_{A}(Y)+C_{B} v_{B}(Y)
$$


where

$$
v_{A}(Y)=\mathcal{W}_{0}(Y) \sim \mathcal{W}_{0}^{\prime}(0) Y+O\left(Y^{2}\right)
$$

and

$$
v_{B}(Y)=\mathcal{W}_{0} \int_{0}^{Y} \frac{d Y}{\mathcal{W}_{0}^{2}} \sim \frac{1}{\mathcal{W}_{0}{ }^{\prime}(0)}+O(Y \log Y)
$$

Observe that

$$
v_{A}(Y) \sim \mathcal{W}_{0}^{\prime}(0) Y+O\left(Y^{2}\right)
$$

and

$$
v_{B}(Y) \sim \frac{1}{\mathcal{W}_{0}^{\prime}(0)}+O(Y \log Y)
$$

as $Y \rightarrow 0$, and

$$
v_{A}(Y) \sim Q_{\mathcal{W}} e^{-\kappa Y}, \quad\left(Q_{\mathcal{W}}=\left(C_{\bar{u}}-C_{\bar{w}}\right) e^{\kappa Y_{\delta}}\right)
$$

and

$$
v_{B}(Y) \sim \frac{1}{Q_{\mathcal{W}}} e^{-\kappa Y},
$$

as $Y \rightarrow \infty$. In view of (11d) and (11e), we must set $C_{B}=0$ to satisfy the surface boundary condition (6a); the leading order eigenfunction is, therefore, given by

$$
v_{0}=\mathcal{W}_{0}(Y)
$$

wherein $C_{A}=1$ without any loss of generality. Note from (11f) that the above solution already satisfies the outer boundary condition (3c) on $v$ because $\hat{\alpha}=1$ has been set to the leading order. The higher order solutions $v_{n}, n=1-4$ are now given by

$$
v_{1}=\alpha_{1} \bar{u}, \quad v_{2}=\alpha_{2} \bar{u}, \quad v_{3}=\alpha_{3} \bar{u},
$$

and

$$
v_{4}=\alpha_{4} \bar{u}+\sec ^{2} \theta_{s w} \mathcal{W}_{0} \int_{0}^{Y} \frac{1}{\mathcal{W}_{0}^{2}} \int_{0}^{Y_{1}} \mathcal{W}_{0}^{2} d Y_{2} d Y_{1}
$$

respectively, and the coefficient of $v_{A}$ has been set equal to zero in each case to satisfy some (arbitrarily chosen) normalization constraint. Because $\bar{u} \rightarrow 1$ as $Y \rightarrow \infty$, none of the solutions from (13a) through (13d) satisfy the original outer boundary condition (3c). Although $v_{1}$ through $v_{3}$ at least retain their $O(1)$ magnitude as $Y \rightarrow \infty$, the second term in $v_{4}$ becomes exponentially large there. For large values of $Y,(13 \mathrm{~d})$ can be rewritten in the form

$$
v_{4}=\alpha_{4} \bar{u}+I_{0}\left(\theta_{s w}, \mathcal{W}_{0}\right) v_{B}(Y)+\sec ^{2} \theta_{s w} \mathcal{W}_{0} \int_{0}^{Y} \frac{1}{\mathcal{W}_{0}^{2}} \int_{\infty}^{Y_{1}} \mathcal{W}_{0}^{2} d Y_{2} d Y_{1},
$$


where the functional $I_{0}$ in the second term is defined as

$$
I_{0}\left(\theta_{s w}, \mathcal{W}_{0}\right)=\sec ^{2} \theta_{s w} \int_{0}^{\infty} \mathcal{W}_{0}^{2} d Y
$$

so that the third term becomes exponentially small as $Y \rightarrow \infty$.

The ordering within the asymptotic series (5b) breaks down when $\beta^{1 / 2} v_{1}$ becomes comparable in magnitude to the leading-order term $v_{0}$ (i.e., when $\mathcal{W}_{0}(Y)=O\left(\beta^{1 / 2}\right)$ ). In view of the assumed behaviour (1a, b), this breakdown occurs when $Y=O\left[-\frac{1}{2 \kappa} \log (\beta)\right]$ (i.e., in the region close to the outer edge of the mean boundary layer). (See figure 1.) The new coordinate is introduced as

$$
\tilde{Y}=Y+\frac{1}{2 \kappa} \log (\beta)=O(1)
$$

which is appropriate for this region. The vertical velocity perturbation $v$ is expanded in the form

$$
v=\beta^{3 / 2} \tilde{v}_{1}(\tilde{Y})+\beta^{2} \tilde{v}_{2}(\tilde{Y})+\beta^{5 / 2} \tilde{v}_{3}(\tilde{Y})+\ldots,
$$

which is suggested by the outer behaviour of the main-deck solutions (12) and (13a-d). If we note that the coefficient function $\mathcal{W}$ expands as

$$
\mathcal{W} \sim \beta^{1 / 2} \tilde{\mathcal{W}}_{1}+\beta \tilde{\mathcal{W}}_{2}+\beta^{3 / 2} \tilde{\mathcal{W}}_{3}+\ldots
$$

in the edge-layer region where

$$
\tilde{\mathcal{W}}_{1}=Q_{\mathcal{W}} e^{-\kappa \bar{Y}}+\alpha_{1} \quad\left(Q_{\mathcal{W}}=\left(C_{\bar{u}}-C_{\bar{w}}\right) e^{\kappa Y_{\delta}}\right)
$$

and

$$
\tilde{\mathcal{W}}_{n}=Q_{\bar{u}} \alpha_{n-1} e^{-\kappa \bar{Y}}+\alpha_{n} \quad \text { for } n>1 \quad\left(Q_{\bar{u}}=C_{\bar{u}} e^{\kappa Y_{\delta}}\right)
$$

then we find that the perturbation quantities $\tilde{v}_{n}, n=1-3$ are governed by the equations

$$
\begin{gathered}
\mathcal{L}_{\tilde{\mathcal{W}}_{1}} \tilde{v}_{1}=0, \\
\mathcal{L}_{\tilde{\mathcal{W}}_{1}} \tilde{v}_{2}=-\alpha_{1} \mathcal{L}_{\tilde{\mathcal{W}}_{2}} \tilde{v}_{1},
\end{gathered}
$$

and

$$
\mathcal{L}_{\tilde{\mathcal{W}}_{1}} \tilde{v}_{3}=-\alpha_{2} \mathcal{L}_{\tilde{\mathcal{W}}_{3}} \tilde{v}_{1}-\alpha_{1} \mathcal{L}_{\overline{\mathcal{W}}_{2}} \tilde{v}_{2}
$$

The operators $\mathcal{L}_{\tilde{W}_{1}}, \mathcal{L}_{\tilde{W}_{2}}$, and $\mathcal{L}_{\bar{W}_{3}}$ are defined analogously to those in $(10 \mathrm{c}, \mathrm{d})$. 
Note that the expansion (16) has been truncated at the third term (i.e., at $O\left(\beta^{5 / 2}\right)$ ) because the latter will match the leading contributions from both $v_{3}$ and $v_{4}$ from the main boundarylayer region. The solutions for the edge-layer perturbations, for which the inner limit as $\tilde{y} \rightarrow \infty$ matches the outer limit of the main-deck solutions (12) and (13a-d), are given by

$$
\begin{gathered}
\tilde{v}_{1}=\tilde{v}_{A}(\tilde{Y}), \\
\tilde{v}_{2}=\tilde{\mathcal{W}}_{2}(\tilde{Y}),
\end{gathered}
$$

and

$$
\tilde{v}_{3}=\tilde{\mathcal{W}}_{3}(\tilde{Y})+C_{3} \tilde{v}_{A}(\tilde{Y})+I_{0} \tilde{v}_{B}(\tilde{Y})
$$

where

$$
\tilde{v}_{A}(\tilde{Y})=\tilde{\mathcal{W}}_{1}(\tilde{Y})
$$

and

$$
\tilde{v}_{B}(\tilde{Y})=\tilde{\mathcal{W}}_{1} \int_{0}^{\tilde{Y}} \frac{d \tilde{Y}}{\tilde{\mathcal{W}}_{1}^{2}}
$$

analogous to $(11 \mathrm{~b}, \mathrm{c}) ; C_{3}$ is a constant that does not need to be determined for this analysis. Because the solutions (19a) through (19c) can also be obtained by expressing the main-deck solution in terms the edge-layer variable $\tilde{Y}$ and reexpanding in powers of $\beta$, the edge layer plays a passive role in determining the overall dynamics of the long-wavelength disturbance.

Because $\tilde{\mathcal{W}}_{1} \rightarrow \alpha_{1}$ as $\tilde{Y} \rightarrow+\infty$, neither $\tilde{v}_{1}, \tilde{v}_{2}$, nor $\tilde{v}_{3}$ satisfy the original outer boundary condition (3c). Moreover, $\tilde{v}_{3}$ exhibits a linear growth

$$
\tilde{v}_{3} \sim \frac{I_{0}}{\alpha_{1}} \tilde{Y} \quad \text { as } \quad \tilde{Y} \rightarrow \infty
$$

which makes the asymptotic series (16) invalid at sufficiently large values of $\tilde{Y}$. This linear growth of the eigenfunction magnitude is cut off within the potential-flow region (or the upper deck), where $Y_{u} \equiv \beta Y=O(1)$. As we will observe below, the leading-order eigenfunction within the potential-flow region has a simple, exponentially decaying character. The zeroth-order (i.e., the constant) term from the small- $Y_{u}$ Taylor expansion of this eigenfunction matches the outer behaviour of $\tilde{v}_{1}$ from the edge-layer region. The coefficient of the linear term from the above Taylor expansion now matches with the slope of the linearly growing component (20) from the edge-layer solution to provide the dispersion relationship for the eigenvalue correction $\alpha_{1}$, which 
determines the spatial growth rate of the long-wavelength crossflow vortex. As seen from (20), the slope of the outer limit of $\tilde{v}_{3}$ is completely determined by the properties of the mean-flow profiles within the main boundary-layer region and does not depend on the assumed form of vorticity decay as $Y \rightarrow \infty$. Thus, the dispersion relationship obtained will actually be valid for a much wider class of boundary-layer profiles.

Only the leading-order solution must be considered within the upper deck region, wherein

$$
\mathcal{W} \sim \beta^{1 / 2} \alpha_{1}+\beta \alpha_{2}+\ldots
$$

and

$$
v \sim \beta^{3 / 2} \breve{v}_{1}+\ldots
$$

The Rayleigh's equation (4a) now reduces to

$$
\frac{d^{2} \breve{v}_{1}}{d Y_{u}^{2}}-\sec ^{2} \theta_{s w} \breve{v}_{1}=0
$$

to the leading order; the solution to (22), which satisfies the outer boundary condition (3c), is given by

$$
\breve{v}_{1}=v_{1}(0) e^{-\sec \theta_{s w} Y_{u}}
$$

A match with the leading-order solution from the edge layer requires that

$$
\breve{v}_{1}(0)=\alpha_{1}
$$

and the linear component of the Taylor expansion of $\breve{v}_{1}$ about $Y_{u}=0$ matches onto (20) provided that

$$
\frac{I_{0}}{\alpha_{1}}=-\sec \theta_{s w} \alpha_{1}
$$

where $I_{0}\left(\theta_{s w}, \bar{u}, \bar{w}\right)$ is defined by $(14 b)$. Equation (25) yields the dispersion relation

$$
\alpha_{1}= \pm i\left(\frac{I_{0}}{\sec \theta_{s w}}\right)^{1 / 2}
$$

If we restore the above result to the streamwise wave number $\alpha$, then we have the asymptotic expansion

$$
\alpha \sim-\beta \tan \theta_{s w} \pm i \beta^{3 / 2} \frac{\sin \theta_{s w}}{\cos ^{3 / 2} \theta_{s w}}\left[\int_{0}^{\infty}(\bar{w}-\bar{u})^{2} d Y\right]^{1 / 2} \text { as } \beta \rightarrow 0
$$


Equation (27) shows that the first correction to the leading-order eigenvalue is purely imaginary. Accordingly, it corresponds to a relatively small rate of amplification or decay along the $X$ direction, depending on a. the $\operatorname{sign}$ of $\sin \theta_{s w}$ and $\mathrm{b}$. whether the minus or the plus sign is chosen in (27). For $\sin \theta_{s w}>0$, the choice of minus sign leads to a spatially unstable normal mode, whereas the plus sign needs to be chosen if $\sin \theta_{s w}<0$. The appearance of two normal modes (both of which have the same wave number in the $X$ direction) is not surprising in view of the asymptotic expansion (4f), which proceeds in integral powers of $\beta^{1 / 2}$. Equation (4f) implies that, even though $d \beta / d \alpha \rightarrow 0$ as $\beta \rightarrow 0, d^{2} \beta / d \alpha^{2}$ becomes singular in this limit. This behaviour explains the observed coalescence of the unstable and stable modes in the long-wavelength limit. The results of figure 2(a) will confirm that the unstable mode does indeed describe the longwavelength behavior of the unstable Rayleigh solution that is obtained numerically, in general. It should be noted that the unstable modes is physically relevant only if it can be realized as the time-asymptotic limit of the corresponding initial boundary value problem. (In other words, if the underlying boundary-layer flow is convective unstable near the location of stability analysis (Huerre and Monkewitz, 1990).) We will assume that the normal mode is physically realistic throughout this paper. See the remarks by Choudhari (1994) in this context.

As is common with long-wavelength instabilities, the amplification rate implied by (27) is independent of the details of the velocity profiles that correspond to the underlying boundarylayer flow and is completely determined by both the local sweep angle (measured relative to the direction of amplification) and the momentum integral associated with the jetlike crossflow profile. A similar result was shown to apply in the case of two-dimensional wakes and jets in the classic paper by Drazin and Howard (1962). They provided a physical explanation for the result with an estimate of the centrifugal force associated with the curved streamline that results from particle displacement induced by the disturbance. The paper by Papageorgiou and Smith (1989) placed Drazin and Howard's analysis in a proper asymptotic framework, and we have shown an analogous structure to be applicable to the stationary crossflow instability of three-dimensional boundary-layer flows. During this process, we have also indicated why the result (27) is independent of the specific form of vorticity decay that is associated with the underlying boundary layer. It is also interesting to note that in spite of the spatial growth rate being asymptotically small with respect to the real part of the wavenumber, the long-wavelength 
inviscid mode does not have a critical layer inside the boundary layer.

Now that a self-consistent solution has been derived for the long-wavelength Rayleigh problem, we can justify why the eigenvalue expansion (4f) was chosen to proceed in powers of $\beta^{1 / 2}$ rather than, for example, in powers of $\beta^{2}$ as a regular perturbation would have indicated. Therefore, suppose that the eigenvalue expansion (4f) proceeds in integral powers of $\beta^{1 / J}$. That is,

$$
\hat{\alpha} \sim-1+\sum_{j=0}^{\infty} \beta^{j / J} \alpha_{j},
$$

where $J$ is an integer to be determined during the course of the analysis. This section demonstrates that the leading term of the corresponding expansion for the eigenfunction is a simple displacement of the jetlike crossflow profile in the wall-normal direction. As indicated by (7b), the first-order correction to this displacement behaviour is caused by the $O\left(\beta^{1 / J}\right)$ deviation in the directional profile $\mathcal{W}$ from its jetlike behaviour $\mathcal{W}_{0}$, which is forced by the eigenvalue correction $\alpha_{1}$ in (28). The discussion that follows (19e) implies that the above correction term can be continued across the edge layer without any change in its order of magnitude and that it rapidly tends to an $O\left(\beta^{1 / J}\right)$ constant near the outer part of the edge layer. On the other hand, the $O\left(\beta^{2}\right)$ correction term, which in part is forced by the operator $\mathcal{M}$ in the Rayleigh's equation, gives rise to the singular component (i.e., $v_{B}$ ) in the eigenfunction. This part exhibits an exponential growth in the main deck, which leads to a magnification by $O\left(\beta^{-1 / 2}\right)$ in its order of magnitude as $Y \rightarrow \infty$. The exponential growth is cut off within the edge layer and is converted to a linear growth that amounts to an additional magnification by $O\left(\beta^{-1}\right)$ in the magnitude of $v_{B}$ as the wall normal location varies across the edge layer. The $O\left(\beta^{2-\frac{1}{2}-1}\right)$ linear component produced in this manner is commensurate with the $O\left(\beta^{\frac{1}{J}}\right)$ constant behaviour at the inner edge of the upper deck only if

$$
\left(2-\frac{1}{J}\right)-1=\frac{1}{M}
$$

(i.e., if $J=2$ as assumed to this point).

To verify the predictions of the long-wavelength asymptotic theory and to estimate the range of wave numbers in which the relevant approximation is reasonable in a numerical sense, the asymptotic predictions based on (27) must be compared with those obtained from a numerical solution of the Rayleigh's equation. Of course, it should be borne in mind that the primary purpose of asymptotics is not to provide numerical results, but to pinpoint the dominant physical 
processes that determine the structure and the growth properties of the unstable disturbance. For convenience, we have opted to illustrate the above comparison in the case of a self-similar FSC profile with the Hartree parameter equal to 0.6 and a local sweep angle of $\theta_{s w}=45^{\circ}$. To enable the reader to form an overall perspective, the results of the Rayleigh calculation throughout the region of instability are shown in figure 2(a). The small wave-number portion of this graph has been replotted in figure 2(b); the additional dashed curve indicates the asymptotic result. Note that in view of the nearly linear behaviour of the Real $(\alpha)$ curve in figure $2(a)$, the corresponding long-wavelength prediction remains accurate throughout the region of crossflow instability. This behaviour is a result of the narrow directionality of the stationary crossflow vortices. On the other hand, the graph for $\operatorname{Imag}(\alpha)$ in figure $2(\mathrm{~b})$ indicates that the spatial amplification rate begins to deviate from its square-root behaviour at small values of the wavenumber parameter $\beta$.

For the self-similar family of FSC boundary layers, the profiles $\bar{u}$ and $\bar{w}$ are dependent only on the Hartree parameter $\beta_{H}$; for $\beta_{H}=0.6$, the normalized crossflow-momentum integral $\int_{0}^{\infty}(\bar{w}-\bar{u})^{2} d Y$ has a value of 0.039 . Note that for the self-similar FSC profile, $Y$ corresponds to the similarity coordinate $y^{*}\left[\mathcal{V}^{*} \cos \theta_{s w} /\left(2-\beta_{H}\right) \nu^{*} x^{*}\right]^{1 / 2}$, i.e., $L^{*} \equiv\left[\mathcal{V}^{*} \cos \theta_{s w} /\left(2-\beta_{H}\right) \nu^{*} x^{*}\right]^{-1 / 2}$. For future reference, figure $2(\mathrm{c})$ shows the variation in the latter quantity as a function of the Hartree parameter $\beta_{H}$.

A similar comparison for the real and imaginary parts of $\alpha$ as functions of the sweep angle $\theta_{s w}$ at a fixed wave number of $\beta=0.01$ is indicated in figure $2(\mathrm{~d})$. Because $\beta=0.01$ represents a small value for the wave number parameter, the asymptotic prediction for Real $(\alpha)$ is virtually indistinguishable from the corresponding numerical result based on the Rayleigh calculation. The graph for $\operatorname{Imag}(\alpha)$ shows that the long-wavelength prediction for the spatial growth rate is satisfactory throughout the range of sweep angles considered; however, a slight loss of accuracy is observed when $\theta_{s w} \rightarrow 90^{\circ}$.

\section{Effect of Convex Streamwise Curvature}

For the analysis in section 2 , we had assumed that the curvature of the body surface was negligible. However, cases of practical interest (Collier and Malik, 1987) exist for which the curvature is strong enough to modify the growth rate of the stationary Rayleigh modes. The effects of sur- 
face curvature are likely to be most significant (in a relative sense) when the associated crossflow instability is weak (i.e., when $\theta_{s w} \rightarrow 0$ ). This limit corresponds to a mean flow that is predominantly along the positive $X$ direction across the entire boundary layer. However, note that the existence of crossflow instability depends on a balance between the streamwise and spanwise convection terms in the governing equations. This balance, which is reflected through the combination $\alpha \cos \theta_{s w} \bar{u}+\beta \sin \theta_{s w} \bar{w}$ in (3a), implies that when $\theta_{s w} \rightarrow 0, \alpha \rightarrow 0$ in such a way that $\hat{\alpha}$ remains finite. A further balance between the convective and the centrifugal terms in the normal-momentum equation (not shown in this paper) indicates that the normal-mode growth begins to be comparably affected by both crossflow and surface curvature terms when the magnitude of the local sweep angle $\theta_{s w}$ has fallen to an $O\left(R^{-1 / 2} \kappa^{1 / 2}\right)$ level. Here, $\kappa \equiv L^{*} / r_{0}^{*}(<<1)$ denotes the nondimensional curvature of the surface based on $r_{0}^{*}$, which is the local radius of curvature along the $X$ direction; we have assumed the other Gaussian curvature of the surface (termed the "transverse" curvature in literature) to be zero for convenience.

The parameter region that involves the combined effects of crossflow and surface curvature may also be approached from the other extreme in which the starting point of the analysis is the Görtler instability of a two-dimensional boundary layer, and the crossflow parameter (i.e., the local sweep angle) is allowed to increase from its initial zero magnitude with the transverse wave number $\beta$ fixed at some $O(1)$ value. This approach was taken by Bassom and Hall (1991a), who extended the pioneering studies of Hall (1982, 1985); Denier, Hall, and Seddougui (1990); and Timoshin (1990) to include three-dimensional boundary layers. As shown by Bassom and Hall, the inviscid instability under the combined effects of weak crossflow and surface curvature is governed by the Rayleigh's equation for streamwise-elongated vortex structures. This equation contains one additional term that characterizes the influence of centrifugal forces associated with the surface curvature. After the introduction of the scaled sweep angle $\theta_{0}=\frac{R}{G^{1 / 2}} \theta_{s w}$, where the Görtler number $G$ is defined as $G \equiv 2 R_{\kappa}(>>1)$, equation (2.8a) of Bassom and Hall (1991a) can be expressed in the form

$$
\left(\mathcal{L}_{\mathcal{W}}+\beta^{2} \mathcal{M}+\frac{\chi \bar{u} \bar{u}^{\prime}}{\mathcal{W} \theta_{0}^{2}}\right) v=0 .
$$

Recall that the operators $\mathcal{L}_{\mathcal{W}}$ and $\mathcal{M}$ in (30a) were defined earlier in (4b) and (4c) and the parameter $\chi$ equal to \pm 1 depending on whether the curvature is concave or convex, respectively.

Because the primary interest of Bassom and Hall's investigation was related to Görtler 
instability, they restricted their calculations to the case of concave surface curvature. Their results confirm the findings of Malik and Poll (1985) and Collier and Malik (1988), which show that this type of curvature has a destabilizing effect overall. Blackaby and Choudhari (1993) focused their interest on the neutral solutions to the Bassom and Hall equation. They considered both concave and convex curvatures and established a connection between the corresponding neutral curves by drawing an analogy to the problem of the stability of stratified shear flows. One of their findings, which is of interest to us in this paper, is the existence of a neutral curve that involves a long-wavelength branch in the case of a convex surface. This study showed that the weakly three-dimensional flow over a convex surface is completely stable when the crossflow magnitude is below a certain critical value. As the crossflow parameter exceeds this critical value, disturbances in a finite band of wave numbers become unstable. The bandwidth of unstable disturbances increases with the amount of crossflow applied. In the limit of large crossflow magnitudes, the right branch of the neutral curve (which bounds the region of instability in the $\beta-\theta_{s w}$ plane) approaches the inflectional neutral mode of the Rayleigh solution (Gregory, Stuart, and Walker, 1955); the left branch tends to the long-wavelength limit $(\beta \rightarrow 0)$ that was considered in section 2 .

We now illustrate a simple modification to the analysis in the previous section to account for the stabilizing effect of convex curvature. This modification yields a simple result for the long-wavelength asymptote of the neutral curve found by Blackaby and Choudhari (1993). As indicated in section 2 , the amplification rate of long-wavelength crossflow vortices is determined by the $O\left(\beta^{2}\right)$ term in equation (4a), which involves the operator $\mathcal{M}$. Clearly, in order for the curvature term to nullify this effect, we must have $\theta_{0} \sim \frac{\theta_{00}}{\beta}\left(\theta_{00}=O(1)\right)$ as $\beta \rightarrow 0$. In that case, the asymptotic expansions for the different subregions involved, as well as the relevant solution procedures, remain completely analogous to those described in section 2 above. The first major difference occurs in solving for $v_{4}$ in the main-deck region, which now satisfies

$$
\mathcal{L}_{\mathcal{W}_{0}} v_{4}=-\alpha_{4} \mathcal{L}_{\bar{u}} v_{0}-\alpha_{3} \mathcal{L}_{\bar{u}} v_{1}-\alpha_{2} \mathcal{L}_{\bar{u}} v_{2}-\alpha_{1} \mathcal{L}_{\bar{u}} v_{3}-\mathcal{M}_{G 0} v_{0} \quad v_{4}(0)=0
$$

instead of $(10 \mathrm{a}, \mathrm{b})$, where

$$
\mathcal{M}_{G 0} v_{0}=-\left(\mathcal{W}_{0}^{2}+\frac{\chi \bar{u} \bar{u}^{\prime}}{\theta_{00}^{2}}\right)
$$


Equations (31a, b) lead to

$$
v_{4}=\alpha_{4} \bar{u}+I_{G}\left(\theta_{00}, \mathcal{W}_{0}\right) v_{B}(Y)+\mathcal{W}_{0} \int_{0}^{Y} \frac{1}{\mathcal{W}_{0}^{2}} \int_{\infty}^{Y_{1}} \mathcal{M}_{G 0} \mathcal{W}_{0} d Y_{2} d Y_{1} \quad\left(I_{G}=\int_{0}^{\infty} \mathcal{W}_{0}^{2} d Y-\frac{1}{\theta_{00}^{2}}\right)
$$

which is analogous to (14a). The solutions in the edge-layer region and the upper deck remain the same, except for the replacement of $I_{G}$ by $I_{0}$. This result leads to the dispersion relation

$$
\alpha_{1}= \pm i I_{G}^{1 / 2}
$$

that determines the amplification rate of the vortex. The neutral curve, which corresponds to the imaginary part of $\alpha_{1}$ equal to zero, is then given by

$$
I_{G}=0
$$

That is,

$$
\theta_{0} \sim \frac{1}{\beta\left(2 \int_{0}^{\infty} \mathcal{W}_{0}^{2} d Y\right)^{1 / 2}} \text { as } \beta \rightarrow 0
$$

Note that the large-crossflow behaviour implied by (34) is more relevant in laminar flow control than the $\theta_{0}=O(1)$ case, in view of the strong three dimensionality that is involved in such applications. A comparison of the analytical result above with the numerically obtained neutral curve by Blackaby and Choudhari (1993) is shown in figure 3. The mean flow chosen for this comparison corresponds to the FSC profile with a Hartree parameter of $\beta_{H}=1 / 2$. (See the paper by Blackaby and Choudhari for details.) Figure 3 shows that the long-wavelength asymptote closely approximates the behaviour of nearly the entire left-hand branch of the neutral stability curve in the $\theta_{0}-\beta$ plane.

\section{Viscous Effects}

\subsection{Viscous-Modified Inviscid Regime}

Now consider the effect of viscosity on the long-wavelength solution derived in the previous subsection. Of particular interest is whether or not the viscous effects can nullify the small inviscid growth rate of the long-wavelength vortices to give rise to a lower branch of the neutral stability curve. The answer to this question has important consequences because transition prediction methods of today utilize the viscous, quasi-parallel framework to determine the local 
amplification rate, which is then integrated from the lower to the upper branch station in order to estimate the location of transition onset (Bushnell and Malik, 1987). In addition, the lower branch properties are particularly important in predicting the receptivity ${ }^{1}$ of the threedimensional boundary layer.

Viscous action within the inviscid regions of $Y=O(1)$ and larger can only modify the vertical velocity perturbation found in section 2.1 through a correction term of $O\left[(\beta R)^{-1}\right]$. However, a much larger correction (of $O\left[(\beta R)^{-1 / 3}\right]$ ) is provided by the viscous sublayer close to the wall, which is required so that the disturbance satisfies the no-slip condition at the surface. The $X$ and $Z$ components of the inviscid slip velocity associated with the main-deck solution (12) are given by

$$
u(0)=i \frac{\bar{u}^{\prime}(0)}{\tan \theta s w}+O\left(\beta^{1 / 2}\right)
$$

and

$$
w(0)=i \bar{w}^{\prime}(0)+O\left(\beta^{1 / 2}\right)
$$

The slip velocity given by $(35 \mathrm{a}, \mathrm{b})$ is brought to zero within the viscous sublayer (or the lower deck), where $Y_{l} \equiv(\beta R)^{-1 / 3} Y=O(1)$. The disturbance quantities within this region are expanded in the form

$$
(u, v, w, p)=\left(U_{0}, \beta(\beta R)^{-1 / 3} V_{0}, W_{0},(\beta R)^{-1 / 3} P_{0}\right)+\ldots
$$

By substituting this expansion into the continuity and momentum equations and allowing $\beta R \rightarrow$ $\infty$ with $Y_{l}=O(1)$, we are led to the linearized three-dimensional boundary-layer equations

$$
\begin{gathered}
i\left(W_{0}-\tan \theta_{s w} U_{0}\right)+\frac{d V_{0}}{d Y_{l}}=0 \\
{\left[i \mathcal{W}_{0}{ }^{\prime}(0) \cos \theta_{s w} Y_{l}\right] U_{0}+\cos \theta_{s w} \bar{u}^{\prime}(0) V_{0}=-i \tan \theta_{s w} P_{0}+\frac{d^{2} U_{0}}{d Y_{l}^{2}}} \\
\frac{d P_{0}}{d Y_{l}}=0
\end{gathered}
$$

and

$$
\left[i \mathcal{W}_{0}^{\prime}(0) \cos \theta_{s w} Y_{l}\right] W_{0}+\sin \theta_{s w} \bar{w}^{\prime}(0) V_{0}=-i P_{0}+\frac{d^{2} W_{0}}{d Y_{l}^{2}}
$$

The term receptivity refers to the various physical mechanisms through which the external disturbances generate the unstable eigenmodes of a boundary-layer flow (Morkovin, 1969). 
The boundary conditions for the lower deck velocities correspond to

$$
U_{0}=V_{0}=W_{0}=0 \quad \text { at } Y_{l}=0
$$

and

$$
U_{0}=u(0), W_{0}=w(0) \quad \text { as } \quad Y_{l} \rightarrow \infty
$$

to match with the inner limits $(35 \mathrm{a}, \mathrm{b})$ of the main-deck solutions.

If the equations $(37 \mathrm{a}-\mathrm{c})$ are solved along with the boundary conditions (38a-e), then the vertical-velocity perturbation $V_{0}$ is given by

$$
V_{0}=\frac{V_{\infty}}{A i^{\prime}(0)}\left[\zeta \int_{0}^{\zeta} A i(\tilde{\zeta}) d \tilde{\zeta}-A i^{\prime}(\zeta)+A i^{\prime}(0)\right],
$$

where $A i$ and $A i^{\prime}$ denote the Airy function of the first kind (Abramowitz and Stegun, 1964) and its derivative, respectively. We have set

$$
V_{\infty}=\frac{3 A i^{\prime}(0) \mathcal{W}_{0}{ }^{\prime}(0)}{\left[i \mathcal{W}_{0}^{\prime}(0) \sin \theta_{s w}\right]^{1 / 3}}, \quad \zeta=\left[i \mathcal{W}_{0}^{\prime}(0) \sin \theta_{s w}\right]^{1 / 3} Y_{l}
$$

where the principal branch has been chosen for the cubic root in the definition of $V_{\infty}$ and $\zeta$ so that as $Y_{l} \rightarrow \infty$

$$
V_{0} \sim \mathcal{W}_{0}{ }^{\prime}(0) Y_{l}+V_{\infty}
$$

The constant term within the solution (40) for the vertical velocity perturbation gives rise to a correction to the main-deck solution (12)-(13d) that is of the form

$$
v_{\text {viscous }}=-\beta(\beta R)^{-1 / 3} V_{\infty} \mathcal{W}_{0}{ }^{\prime}(0) v_{B}(Y) .
$$

This viscous correction first affects the spatial growth rate of the long-wavelength vortex when it becomes comparable to the $\beta^{3} v_{4}$ inviscid term, which (shown in section 2) determines the inviscid amplification rate by producing a similar $v_{B}$ component as $Y \rightarrow \infty$. The viscous and inviscid contributions to the coefficient of the $v_{B}$ component are of the same order of magnitude when

$$
\beta=R^{-1 / 7} \beta_{0} \quad\left(\beta_{0}=O(1) \quad \text { as } \quad R \rightarrow \infty\right),
$$

in which case the dispersion relationship (27) is altered to

$$
\alpha \sim-R^{-1 / 7} \beta_{0} \tan \theta_{s w} \pm i R^{-3 / 14} \frac{\sin \theta_{s w}}{\cos ^{1 / 2} \theta_{s w}}\left[\beta_{0}^{3} I_{0}-\beta_{0}^{2 / 3} V_{\infty} \mathcal{W}_{0}{ }^{\prime}(0)\right]^{1 / 2}
$$


Because $V_{\infty}$ is a complex constant, the viscous effects not only modify the inviscid growth rate behaviour but also introduce an $O\left(R^{-3 / 14}\right)$ correction to the inviscid result indicated by the leading term in $(42 \mathrm{~b})$. As $\beta_{0} \rightarrow \infty$, the viscous effects become weaker relative to the inviscid contribution, and we recover the earlier result $(27)$. On the other hand, as $\beta_{0} \rightarrow 0$, the viscous term dominates the eigenvalue correction to yield

$$
\alpha \sim-R^{-1 / 7} \beta_{0} \tan \theta_{s w} \pm i R^{-3 / 14} \beta_{0}^{1 / 3} \frac{\sin \theta_{s w}}{\cos ^{1 / 2} \theta_{s w}}\left[-V_{\infty} \mathcal{W}_{0}^{\prime}(0)\right]^{1 / 2}
$$

With the proper choice of sign for the second term on its right-hand side, eq. (43) yields a vortex mode that amplifies in the $X$ direction, but at a growth rate that is relatively small in comparison with the real part of $\alpha$. However, because the rate of decrease in the growth rate as $\beta_{0} \rightarrow 0$ is significantly slower than that of the real part of $\alpha$, the eigenvalue-correction term in (43) will eventually become as large as the leading term. This indicates that a different asymptotic structure must be considered at this stage. Not surprisingly, the onset of this new

asymptotic structure corresponds to $\beta_{0}=O\left(R^{-3 / 28}\right)$ or, equivalently, $\beta=R^{-1 / 4} \beta_{00}\left[\beta_{00}=O(1)\right]$, which is the triple-deck scaling.

\subsection{Viscous-Inviscid Interactive Regime}

For $\beta=R^{-1 / 4} \beta_{00}$, the disturbance structure is fully viscous-inviscid interactive in nature, and the perturbation quantities within the lower deck expand in the form

$$
(u, v, w, p)=\left(U_{0}, R^{-1 / 2} V_{0}, W_{0}, R^{-1 / 4} P_{0}\right)+\ldots
$$

This expansion leads to the following set of governing equations after substitution into the continuity and momentum equations:

$$
\begin{gathered}
i\left(\alpha_{00} U_{0}+\beta_{00} W_{0}\right)+\frac{d V_{0}}{d Y_{00}}=0, \\
i\left[\alpha_{00} \cos \theta_{s w} \bar{u}^{\prime}(0)+\beta_{00} \sin \theta_{s w} \bar{w}^{\prime}(0)\right] Y_{00} U_{0}+\cos \theta_{s w} \bar{u}^{\prime}(0) V_{0}=-i \alpha_{00} P_{0}+\frac{d^{2} U_{0}}{d Y_{l}^{2}}, \\
\frac{d P_{0}}{d Y_{00}}=0, \\
i\left[\alpha_{00} \cos \theta_{s w} \bar{u}^{\prime}(0)+\beta_{00} \sin \theta_{s w} \bar{w}^{\prime}(0)\right] Y_{00} W_{0}+\sin \theta_{s w} \bar{w}^{\prime}(0) V_{0}=-i \beta_{00} P_{0}+\frac{d^{2} W_{0}}{d Y_{l}^{2}} .
\end{gathered}
$$


Here,

$$
Y_{00}=R^{-1 / 4} y
$$

is the new lower deck coordinate;

$$
\beta_{00} \equiv R^{1 / 4} \beta
$$

is the scaled (i.e., $O(1)$ ) wave-number parameter; the corresponding, scaled eigenvalue $\alpha_{00}$ is defined as

$$
\alpha_{00}=R^{1 / 4} \alpha
$$

The boundary conditions associated with (45a-d) are given by the no-slip conditions

$$
U_{0}=V_{0}=W_{0}=0 \text { at the surface } Y_{00}=0 \text {, }
$$

with the requirements

$$
U_{0} \sim \cos \theta_{s w} \bar{u}^{\prime}(0) A, W_{0}=\sin \theta_{s w} \bar{w}^{\prime}(0) A \quad \text { as } \quad Y_{00} \rightarrow \infty
$$

for matching with the inner limits of the main-deck solutions. The displacement-thickness perturbation $A$ in $(46 \mathrm{~d}, \mathrm{e})$ is related to the pressure perturbation $P_{0}$ via the interactive relationship

$$
P_{0}=\frac{\left(\alpha_{00} \cos \theta_{s w}+\beta_{00} \sin \theta_{s w}\right)^{2}}{\left(\alpha_{00}^{2}+\beta_{00}^{2}\right)^{1 / 2}} A .
$$

The triple-deck problem defined by (45a-d) and (46a-f) is equivalent to the zero-frequency limit of the problem considered by Smith and Stewart (1987) who analyzed the effect of crossflow on the behaviour of Tollmien-Schlichting (TS) instability. Accordingly, the dispersion relation for the interactive stationary vortices can be easily recovered by setting the frequency parameter equal to zero in equation (B1) of Smith and Stewart. This relation is given by

$$
\left(\alpha_{00}^{2}+\beta_{00}^{2}\right)^{1 / 2}\left(\alpha_{00} \cos \theta_{s w}+\beta_{00} \sin \theta_{s w}\right)^{2}+3 A i^{\prime}(0)\left\{i\left[\alpha_{00} \cos \theta_{s w} \bar{u}^{\prime}(0)+\beta_{00} \sin \theta_{s w} \bar{w}^{\prime}(0)\right]\right\}^{5 / 3}=0 .
$$

The dependence of the normal-mode properties on the shape of the base velocity profile has disappeared completely because the leading order eigenvalue $\alpha_{00}$ can be determined with a knowledge of just the wall-shear components $\bar{u}^{\prime}(0)$ and $\bar{w}^{\prime}(0)$.

Two of the roots of the dispersion relation (44) behave in accordance with

$$
\alpha_{00} \sim-\beta_{00} \tan \theta_{s w} \pm i \beta_{00}^{1 / 3} \frac{\sin \theta_{s w}}{\cos ^{1 / 2} \theta_{s w}}\left[-V_{\infty} \mathcal{W}_{0}{ }^{\prime}(0)\right]^{1 / 2} \quad \text { when } \quad \beta_{00} \rightarrow \infty
$$


Hence, these match onto the stable and unstable modes from equation (43) when $1<<\beta_{00}<<$ $R^{3 / 28}$. On the other hand, numerical calculations (not shown in this paper) have revealed that as $\beta_{00} \rightarrow 0$ the analytical continuation of the unstable mode in (47b) behaves in the manner

$$
\alpha_{00} \sim-\beta_{00} \tan \theta_{w}-i \beta_{00}^{g / 5} \frac{\left|\cos \theta_{s w}-\tan \theta_{w} \sin \theta_{s w}\right|^{6 / 5}}{\lambda\left[-3 A i^{\prime}(0)\right]^{3 / 5} \cos ^{8 / 5} \theta_{w}}
$$

where $\theta_{w} \equiv \arctan \left[\tan \theta_{s w} \frac{\bar{w}^{\prime}(0)}{\bar{u}^{\prime}(0)}\right]$ and $\lambda \equiv\left[\cos ^{2} \theta_{s w} \bar{u}^{\prime}(0)^{2}+\sin ^{2} \theta_{s w} \bar{w}^{\prime}(0)^{2}\right]^{1 / 2}$ define the direction and the magnitude, respectively, of the wall shear stress vector $\left[\cos \theta_{s w} \bar{u}^{\prime}(0), \sin \theta_{s w} \bar{w}^{\prime}(0)\right]$. Equation (47c) implies that the mode that is unstable at large values of $\beta_{00}$ also remains unstable at very small values of $\beta_{00}$, although the spatial growth rate decreases at a faster than linear rate when $\beta_{00} \rightarrow 0$. We also made an attempt to solve the dispersion relation (47a) numerically in order to track the behaviour of the stable mode in $(47 \mathrm{~b})$ across the $\beta_{00}=O(1)$ range and into the $\beta_{00}<<1$ regime; however, in each case, the Newton-Raphson method failed to converge after $\beta_{00}$ became sufficiently small. Consequently, the behaviour of the stable mode could not be determined either analytically or numerically.

It is worth noting that the large wave-number limit implied by (47b) also represents nearly neutral vortex modes (as in the small wave-number limit (47c)). However, the large wave-number modes are aligned with the local free-stream direction, whereas the small wave-number modes of (47c) are aligned with the local wall-shear direction. Substitution of (47c) into the momentum equations $(45 \mathrm{~b}, \mathrm{~d})$ implies that the convection term associated with the linear behaviour of the local directional mean profile tends to zero as $\beta_{00} \rightarrow 0$. Of course, this decrease in the magnitude of the convection term cannot continue indefinitely because when $\beta_{00}=O\left(R^{-5 / 28}\right)$, or

$$
\beta=R^{-3 / 7} \tilde{\beta}, \quad(\tilde{\beta}=O(1)),
$$

one must consider the more refined approximation for the mean-flow profiles

$$
\bar{u} \sim \bar{u}_{1} Y+\bar{u}_{2} Y^{2}+\ldots \quad(\text { as } Y \rightarrow 0)
$$

and

$$
\bar{w} \sim \bar{w}_{1} Y+\bar{w}_{2} Y^{2}+\ldots \quad(\text { as } Y \rightarrow 0)
$$

rather than the linear approximation that has been sufficient thus far. For brevity of notation, we have denoted the wall-shear components $\bar{u}^{\prime}(0)$ and $\bar{w}^{\prime}(0)$ by $\bar{u}_{1}$ and $\bar{w}_{1}$, respectively, in equations $(48 \mathrm{~b}, \mathrm{c})$. 
The solutions for the lower deck perturbations in (44) suggest that as $\beta_{00} \rightarrow 0$ the component of the horizontal velocity perturbation along the direction of the wave-number vector (i.e., the component that is proportional to the divergence of the horizontal velocity field $\left.\left(U_{0}, W_{0}\right)\right)$ becomes asymptotically small in comparison with the component that is orthogonal to the wave-number vector (and, hence, is proportional to the vertical vorticity field). However, the dispersion relation for the unstable crossflow mode is still determined by the former component which, along with the perturbations in vertical velocity and pressure, is decoupled from the vertical vorticity field. In view of this decoupling, we limit our attention to the former set of quantities (in particular, $V_{0}$ and $P_{0}$ ) for $\beta<<R^{-1 / 4}$. The vertical vorticity field and the dominant velocity perturbations can be evaluated on an a posteriori basis, if necessary.

\subsection{Interactive Wall-Shear-Aligned Vortices}

For $\beta=O\left(R^{-3 / 7}\right)$, a modified interactive structure is obtained for which the eigenvalue $\alpha$ and the lower deck perturbations $(U, V, W, P)$ expand as

$$
\alpha \sim-R^{-3 / 7} \tilde{\beta} \tan \theta_{w}+R^{-4 / 7} \tilde{\alpha}_{1}
$$

and

$$
(u, v, w, p)=\left(U_{0}, R^{-5 / 7} V_{0}, W_{0}, R^{-3 / 7} P_{0}\right)+\ldots
$$

respectively; the new lower deck coordinate is

$$
\tilde{Y}=R^{1 / 7} Y
$$

The momentum equation along $\tilde{Y}$ reduces to a form that is analogous to (37c) and (45c). After a suitable manipulation of the continuity equation and the momentum equations along the $X$ and $Z$ directions, one finds that $V_{0}$ is governed by the third-order equation

$$
\frac{d^{3} V_{0}}{d \tilde{Y}^{3}}-i\left(\tilde{\lambda} \tilde{Y}+\tilde{\Lambda} \tilde{Y}^{2}\right) \frac{d V_{0}}{d \tilde{Y}}+i(\tilde{\lambda}+2 \tilde{\Lambda} \tilde{Y}) V_{0}=\tilde{\beta}^{2} \sec ^{2} \theta_{w} P_{0}
$$

and is subject to the wall boundary conditions

$$
V_{0}(0)=\frac{d V_{0}(\tilde{Y}=0)}{d \tilde{Y}}=0
$$

and the condition

$$
\frac{d V_{0}}{d \tilde{Y}} \sim \quad-i(\tilde{\lambda}+2 \tilde{\Lambda} \tilde{Y}) A \quad \text { as } \quad \tilde{Y} \rightarrow \infty
$$


for matching of $V_{0}$ with the main deck solution

$$
v=-i R^{-3 / 7}\left[\tilde{\beta}\left(\sin \theta_{s w} \bar{w}-\tan \theta_{w} \cos \theta_{s w} \bar{u}\right)+R^{-1 / 7} \tilde{\alpha}_{1} \cos \theta_{s w} \bar{u}\right] A
$$

Here,

$$
\tilde{\lambda}=\tilde{\alpha}_{1} \cos \theta_{s w} \bar{u}_{1}, \quad \tilde{\Lambda}=\tilde{\beta}\left(\sin \theta_{s w} \bar{w}_{2}-\tan \theta_{w} \cos \theta_{s w} \bar{u}_{2}\right)
$$

and the displacement thickness perturbation $A$ is related to the pressure perturbation $P_{0}$ via the interactive relation

$$
P_{0}=\tilde{\beta} \cos \theta_{w}\left(\sin \theta_{s w}-\tan \theta_{w} \cos \theta_{s w}\right)^{2} A
$$

which is obtained from (46f) and (49a). Note that the set of equations (50) through (51c) can be simplified through an affine transformation so that the independent parameter $\tilde{\Lambda}$ appears only in (51c). However, the advantage gained through this procedure is relatively small; hence, we will not introduce this transformation in the present analysis.

For a given value of $\tilde{\beta}$, solutions to the inhomogeneous boundary value problem (50) and $(51 \mathrm{a}-\mathrm{c})$ are possible only for a limited set of values for $\tilde{\lambda}$, each of which determines the growth or decay rate of the respective eigenmode in view of the definition (5le) of $\tilde{\lambda}$. In the limit of $\tilde{\beta} \rightarrow \infty, \tilde{\alpha}_{1}$ increases at a faster than linear rate (see (45b)). Therefore, the quadratic terms in (50) become relatively small, thereby yielding the required overlap with the governing equations (37) and (38) in the triple-deck regime. On the other hand, when $\tilde{\beta}$ is reduced, the growth rate (as well as the real part of $\tilde{\alpha}_{1}$ ) is expected to decrease monotonically; therefore, the linear convection term in (50) that involves $\tilde{\lambda}$ will diminish in importance in comparison with the quadratic part. As a result, the eigenvalue parameter $\tilde{\lambda}$ will drop out of the leading-order equation for $V_{0}$. The latter does not contain a pressure-gradient term and is identically satisfied by the small- $Y$ behaviour of the main deck solution $(51 \mathrm{~d})$ :

$$
v \sim-i R^{-3 / 7} \tilde{\Lambda} Y^{2} A
$$

which also satisfies the boundary conditions (51a-c) above. The correction to this inviscid solution is driven by both the residual convective acceleration that involves $\tilde{\lambda}$ and the inviscid solution (52), and also by the pressure-gradient term in the original governing equation (50). A balance of the two forcing terms provides the asymptotic scaling $\tilde{\lambda}=O\left(\tilde{\beta}^{5 / 2}\right)$ for the eigenvalue 
parameter $\tilde{\lambda}$ in the limit $\tilde{\beta} \rightarrow 0$. Accordingly, we introduce the rescaled eigenvalue

$$
\tilde{\tilde{\lambda}}=\tilde{\beta}^{-5 / 2} \tilde{\lambda},
$$

the rescaled wall-layer coordinate

$$
\tilde{\tilde{Y}}=\tilde{\beta}^{1 / 4} \tilde{Y}
$$

and the scaled pressure-gradient parameter

$$
\tilde{\tilde{\Lambda}}=\tilde{\beta}^{-1} \tilde{\Lambda}=\sin \theta_{s w} \bar{w}_{2}-\tan \theta_{w} \cos \theta_{s w} \bar{u}_{2}
$$

We expand $V_{0}$ and $P_{0}$ in the forms

$$
V_{0}=-\tilde{\beta}^{1 / 2} i \tilde{\tilde{\Lambda}} \tilde{\tilde{Y}}^{2} A+\tilde{\beta}^{9 / 4} V_{01}+\ldots
$$

and

$$
P_{0}=\tilde{\beta} P_{01}+\ldots
$$

as $\tilde{\beta} \rightarrow 0$, where $V_{01}$ is now a function of $\tilde{\tilde{Y}}$. If equations (53a-e) are substituted into (50) through (51c), $V_{01}$ is governed by the equation

$$
\frac{d^{3} V_{01}}{d \tilde{\tilde{Y}}^{3}}-i \tilde{\tilde{\Lambda}} \tilde{\tilde{Y}}^{2} \frac{d V_{01}}{d \tilde{\tilde{Y}}}+2 i \tilde{\tilde{\Lambda}} \tilde{\tilde{Y}} V_{01}=f(\tilde{\tilde{Y}}) \quad\left[f(\tilde{\tilde{Y}})=\sec ^{2} \theta_{w} P_{01}+\tilde{\tilde{\lambda}} \tilde{\tilde{\Lambda}} \tilde{\tilde{Y}}^{2} A\right]
$$

subject to the boundary conditions

$$
V_{01}(0)=\frac{d V_{01}(\tilde{\tilde{Y}}=0)}{d \tilde{\tilde{Y}}}=0
$$

and

$$
\frac{d V_{01}}{d \tilde{\tilde{Y}}} \sim-i \tilde{\tilde{\lambda}} A \quad \text { as } \quad \tilde{\tilde{y}} \rightarrow \infty
$$

and the interactive relation

$$
P_{01}=\cos \theta_{w}\left(\sin \theta_{s w}-\tan \theta_{w} \cos \theta_{s w}\right)^{2} A .
$$

Solutions to this inhomogeneous boundary value problem are possible only if the eigenvalue correction $\tilde{\tilde{\lambda}}$ satisfies the solvability condition

$$
\int_{0}^{\infty} f F d \tilde{\tilde{Y}}=0
$$


where $F$ represents the eigensolution of the adjoint problem

$$
\frac{d^{3} V_{01}}{d \tilde{\tilde{Y}}^{3}}-i \tilde{\tilde{\Lambda}} \tilde{\tilde{Y}}^{2} \frac{d V_{01}}{d \tilde{\tilde{Y}}}-4 i \tilde{\tilde{\Lambda}} \tilde{\tilde{Y}} V_{01}=0 ; \quad F(0)=0, \quad \frac{d F}{d \tilde{\tilde{Y}}}, \frac{d^{2} F}{d \tilde{\tilde{Y}}^{2}} \rightarrow 0 \quad \text { as } \quad \tilde{\tilde{Y}} \rightarrow \infty . \quad(56 a, b, c)
$$

We can show that $F$ is given by

$$
F=s U(0, s)
$$

within a constant multiplicative coefficient; here,

$$
s=(4 i \tilde{\tilde{\Lambda}})^{1 / 4} \tilde{\tilde{Y}}
$$

(with the assumption that the principal branch has been chosen for the multivalued function on the right-hand side) and $U(0, s)$ denotes the parabolic cylinder function (Abramowitz and Stegun, 1964). Based on the property that $\int_{0}^{\infty} s^{3} U(0, s)=U(0,0)$, the dispersion relation in the $\tilde{\beta}<<1$ regime is given by

$$
\tilde{\tilde{\lambda}}=-\sec \theta_{w}\left(\sin \theta_{s w}-\tan \theta_{w} \cos \theta_{s w}\right)^{2}\left[\frac{\int_{0}^{\infty} s U(0, s) d s}{U(0,0)}\right] \frac{(i \tilde{\tilde{\Lambda}})^{1 / 2}}{2 \tilde{\tilde{\Lambda}}} .
$$

The numerical value of the bracketed ratio (which involves parabolic cylinder functions) on the right-hand side of (57c) was quoted by Hall (1986) to be 1.198 , approximately, and our calculations verified this result. In view of (49a), (51e), and (53a), equation (57c) implies a positive growth rate for the crossflow vortex under consideration. Equation $(57 \mathrm{c})$ is, in fact, identical to the dispersion relation obtained by Hall (1986) in the context of a rotating-disk flow, provided that the Coriolis terms are set equal to zero in the latter equation.

Equations (53a) and (57c) imply that for $R^{-\tau}<<\tilde{\beta}<<1(\tau>0)$ the spatial growth rate of the crossflow vortex, as well as the wave-number correction given by the real part of $\tilde{\alpha}_{1}$, decreases at a rate that is proportional to $\tilde{\beta}^{5 / 2}$. A comparison of $(57 \mathrm{c})$ with $(48 \mathrm{~b})$ reveals that this rate of decrease is significantly greater than the corresponding decrease (shown in section 4.2) when $\beta$ was reduced below the range $\beta=O\left(R^{-1 / 4}\right)$, which corresponds to the triple-deck regime. This decrease in $\tilde{\alpha}_{1}$ is accompanied by a corresponding decrease in the wave-number-aligned component of the disturbance slip velocity at the inner edge of the main deck. (See (54d).) Note in (5la) through (54e) that this latter component gives rise (through viscous retardation) to the displacement perturbation $A$ within the lower deck. The displacement perturbation induces a pressure disturbance in the upper deck that is fed back to the lower deck, which 
sustains the interactive structure of the disturbance. As a consequence of the decrease in this slip-velocity component as $\tilde{\beta}$ decreases, the higher order corrections (which result from the previously neglected cubic and higher order derivatives of the directional mean profile near the surface) exert an increasing influence on the outer behaviour of the correction term $V_{01}$ within the lower deck. For instance, when $\tilde{\beta}=O\left(R^{-1 / 14}\right)$ (i.e., $\beta=O\left(R^{-1 / 2}\right)$ ), the boundary condition (54d) must include the effect of the cubic term in the small- $Y$ Taylor expansion of the main-deck solution (51d). (See Hall, 1986.) Unlike the inviscid leading-order solution in (53d) for which the viscous-diffusion term is equal to zero, the inviscid contribution associated with the cubic term does not satisfy the viscous lower deck equation. The viscous correction to this inviscid contribution would modify the viscous displacement function $A$ by an $O(1)$ amount and hence, also, the dispersion relation $(57 \mathrm{c}$ ) that determines the growth rate of the vortex. However, by using the mean boundary-layer equations, the third-order derivatives of $\bar{u}$ and $\bar{w}$ can be shown to be equal to zero for a large class of boundary-layer flows, such as boundary layers with no suction at the surface. In that case, the dispersion relation $(57 \mathrm{c})$ will remain valid until $\tilde{\beta}=O\left(R^{-8 / 63}\right)$ (i.e., $\beta=O\left(R^{-5 / 9}\right)$ ), at which stage the fourth-order derivative of the directional mean profile $\left(=24\left(\sin \theta_{s w} \bar{w}_{4}-\tan \theta_{w} \cos \theta_{s w} \bar{u}_{4}\right)\right)$ will come into play.

The cubic (or quartic) terms can reduce the growth rate of the crossflow mode to negative values in the $\beta=O\left(R^{-1 / 2}\right.$ ) (or $\beta=O\left(R^{-5 / 9}\right)$ ) regime and cut off the crossflow instability at sufficiently small wave numbers. However, at least for the rotating-disk case Hall's analysis (Hall, 1986) indicates that these terms do not significantly affect the stability properties. Although these aspects are interesting and important in regard to their influence on the receptivity properties (section 5), we will not address them in this paper.

\section{Concluding Remarks}

The stability properties of long-wavelength stationary crossflow modes were examined, beginning with the long-wavelength Rayleigh regime $R^{-\tau}<\beta \beta<1(\tau>0)$. In the latter limit, the leading-order solution to Rayleigh's equation reduces to a simple, vertical displacement of the jetlike crossflow profile. A nominally $O\left(\beta^{2}\right)$ correction term (that involves the operator $\mathcal{M}$ in Rayleigh's equation (4a)) gives rise to a significantly larger amplification rate, specifically $O\left(\beta^{1 / 2}\right)$ relative to the real part of the wave number $\alpha$. This effect is caused by a commensurate 
magnification across the boundary layer in the magnitude of the singular homogeneous solution (viz., $v_{B}$ ) to the long-wavelength form of Rayleigh's equation. The expression for the leadingorder growth rate resembles to an analogous expression derived by Drazin and Howard (1962) for the sinuous mode of a symmetric jet or wake profile. The reason for this similarity is that in both cases the above-mentioned displacement solution satisfies both of the imposed boundary conditions. The most striking difference between the long-wavelength stability properties of the boundary-layer flows considered in this paper and the free-shear flows considered by Drazin and Howard (1962) results from the earlier onset of viscous effects in the boundary-layer case. In particular, as $\beta$ is reduced below the long-wavelength Rayleigh regime, the viscous effects lead to a cascade of asymptotic subregimes across which the orientation of the crossflow vortices changes from the direction of the local inviscid streamline to that of the mean wall-shear vector. The variation in the spatial amplification rate as $\beta$ varies through the subregimes is shown schematically in Figure 4.

The study of the effect of surface curvature in section 3 demonstrates that the convexity of the surface along the longitudinal direction can stabilize the inviscid crossflow modes. A similar effect can be expected in the viscous regimes as well. The influence of convex surface curvature is particularly prominent near the nose of an airfoil and, indeed, has been noted in a number of investigations including those by Malik and Poll (1985) and Collier and Malik (1987).

We now close the discussion by commenting on other possible extensions and applications of the present theory. One important extension of these results is the consideration of compressible base flows and nonzero disturbance frequencies. Both generalizations require relatively minor modifications to the theoretical framework of sections 2 through 4 ; however, the modifications promise results of considerable practical importance. In particular, the inclusion of a nonzero frequency parameter would indicate how a lower branch of the neutral stability curve emerges in the wave-number regime studied herein as the frequency parameter increases to the TollmienSchlichting (TS) regime considered by Stewart and Smith (1988). The development of this analytical connection between crossflow and the TS modes as the Mach number is increased to supersonic values will be interesting. The TS instability of three-dimensional supersonic boundary layers has previously been considered by Choudhari and Streett (1990) and in further detail by Ryzhov and Terent'ev (1991). Other relevant works on unsteady crossflow vortices 
include the paper by Bassom and Gajjar (1988) on high-frequency modes and the work of Balakumar, Hall, and Malik (1991) on the algebraic growth of low-frequency modes in a rotatingdisk boundary layer. A comparison of the results of the abovementioned extension with the results obtained by these authors will be useful. The effects of lateral and longitudinal curvatures in the supersonic regime may also be studied by combining the present analysis with that of Duck and Hall (1989) and Dando (1992), respectively.

Another extension of the present study that we are currently pursuing concerns the wavenumber regime of $\beta \leq O\left(R^{-1 / 2}\right)$, where a lower branch of the neutral stability curve may exist for stationary-vortex instabilities. The possible existence of a lower branch of the neutral stability curve for stationary crossflow vortices on a relatively flat, nonrotating surface has important practical ramifications, in particular for receptivity (Choudhari, 1994). The absence of a lower branch would imply that the induction of crossflow vortices near the leading-edge region (whether by surface disturbances or by free-stream modulations) is likely to be the most important source, overall, of stationary vortex modes in a swept-wing boundary layer. Secondly, the nature of the receptivity caused by nonlocalized, stationary surface roughness will be considerably different from a similar mechanism in the case of nonstationary modes (Choudhari, 1993). Choudhari (1993) showed that the receptivity due to an elongated region of small-amplitude surface nonuniformities can be evaluated as an integral over each infinitesimal subregion. In each of these subregions, a suitable extension of Goldstein (1985) and Ruban's (1985) theory can be applied to predict the local receptivity. When the neutral stability curve possesses a lower branch, the behavior of this integral is similar to that of a standard Laplace integral if the distribution of surface nonuniformities is periodic. The saddle point of the above integral coincides with an interior location that corresponds to a near resonance between the wave numbers of the periodic forcing and the instability mode (Zavol'skii et al. 1983). Thus, the most significant receptivity is produced by a narrow range of locations in the vicinity of this saddle point. In the absence of a lower neutral branch, on the other hand, the above integral becomes dominated by end-point contributions; this indicates that the receptivity is dominated by the far-upstream nonuniformities. Thus, the dependence of receptivity on the roughness geometry in this case must be compared with the simple behaviour observed by Zavol'skii et al. (1983) and Choudhari (1993, 1994). Finally, we note that the efficiency of receptivity caused by a localized 
roughness element in the $\beta<<R^{-1 / 4}$ regime is also expected to be quite different from the analogous localized receptivity problem in the case of the TS waves studied by Goldstein (1985) and Ruban (1985). This difference is because a small-amplitude roughness element produces very little disturbance in the vicinity of the direction that is orthogonal to the wall-shear-stress vector and the latter direction coincides with the direction of the wave-number vector for the crossflow-vortex modes in the above wave-number regime.

In section 4 , we indicated how the dispersion relation from one asymptotic regime matches onto the appropriate limit of the dispersion relation from an adjacent region when the relevant wave-number parameter is allowed to either decrease to zero or tend toward infinity. Physically, this matching procedure is equivalent to considering a fixed location on the body surface and varying the wavelength of the crossflow disturbance. This procedure was done partly for want of a convenient physical model for a three-dimensional boundary layer; however, in practice, a more relevant approach may be to explore the results of an analogous matching procedure in which the disturbance wavelength is kept fixed but the Reynolds number is varied by moving toward the nose region along the body surface (so that the mean boundary-layer profiles must also vary accordingly). Because the long-wavelength modes are nearly neutral (except within the triple-deck regime), it will be useful to examine whether any modulations of the incoming flow along the $z$ direction can directly excite the unstable vortex modes near the nose of the body. Problems of this type have not yet been studied in the context of three-dimensional boundary layers, although some interesting results have been reported for the case of a two-dimensional boundary layer by Goldstein, Leib, and Cowley (1992) and Goldstein and Leib (1993a,b). A similar receptivity problem that involves the generation of Görtler vortices has been studied by Hall (1990).

Great strides have been made recently in the analytical description of the nonlinear stage during the transition process. (Sec, for example, the milestone works by Hall and Smith (1991), Goldstein and Leib (1988), and Goldstein and Choi (1990).) However, with the exception of a recent effort by Bassom and Hall (1991b), not much research has been devoted to the nonlinear development of crossflow-vortex instabilities. Indeed, because the initial amplitudes of stationary crossflow vortices can at times be much larger than those of nonstationary modes (Choudhari and Streett, 1990), the nonlinearity is likely to significantly influence the development of the 
stationary vortices much earlier than in the case of the nonstationary vortices. Because the amplification rates for the stationary vortices remain relatively small in a significant part of the long-wavelength regime, the nonlinear effects might have an influence within the same regime. The likelihood of this possibility in flows of practical interest must be determined.

\section{Acknowledgments}

Financial support for this work was provided by the Theoretical Flow Physics Branch, Fluid Mechanics Division, NASA Langley Research Center, Hampton, VA 23681-0001 under contract number NAS1-19299. The author is grateful to Dr. Marvin Goldstein for some useful comments on the inviscid results. In addition, the author would like to thank Ms. Jonay Campbell for editing this manuscript and Ms. Pamela Chesmar for preparing figures 1 and 4.

\section{References}

[1] Abramowitz, M. and Stegun, I., "Handbook of Mathematical Functions," Dover, New York, 1964.

[2] Balakumar, P., Hall, P., and Malik, M. R., "On the Receptivity and Nonparallel Stability of Travelling Disturbances in Rotating Disk Flow," Theor. Comp. Fluid Dyn., Vol. 3, No. 3, pp. 125-140, 1991.

[3] Bassom, A. B., and Gajjar, J. S. B., "Nonstationary Crossflow Vortices in ThreeDimensional Boundary Layer Flows," Proc. Roy. Soc. Lond. A, Vol. 417, pp. 179-212, 1988.

[4] Bassom, A. P., and Hall, P., "Vortex Instabilities in 3D Boundary Layers: The Relationship Between Görtler and Crossflow Vortices," J. Fluid Mech. Vol. 232, pp. 647-680, 1991.

[5] Bassom, A. P., and Hall, P., " Nonlinear Crossflow Vortices," J. Fluid Mech. Vol. 232, pp. $647-680,1991$.

[6] Bushnell, D. M. and Malik, M. R., "Application of stability theory to laminar flow control Progress and Requirements," Proc. Symp. on the Stability of Time Dependent and Spatially Varying Flows, eds. D. L. Dwoyer and M. Y. Hussaini, Springer, New York, pp. 1-17, 1987. 
[7] Blackaby, N. D., and Choudhari, M., "Inviscid Vortex Motions in weakly Three-Dimensional Boundary Layers and their Relation with Instabilities in Stratified Shear Flows," Proc. Roy. Soc. London A, Vol. 440, pp. 701-710, 1993.

[8] Choudhari, M., "Boundary-Layer Receptivity due to Distributed Surface Imperfections of a Deterministic or Random Nature," Theor. and Comp. Fluid Dyn., Vol. 4, No. 3, pp. 101-118, Feb. 1993 (also: NASA CR-4439, May 1992).

[9] Choudhari, M., "Roughness-Induced Generation of Crossflow Vortices in ThreeDimensional Boundary Layers," Theor. and Comp. Fluid Dyn., Vo. 5, pp. 1-31, Feb. 1994.

[10] Choudhari, M., and Streett, C. L., "Boundary Layer Receptivity Phenomena in ThreeDimensional and High-Speed Boundary Layers," AIAA Paper 90-5258, Oct. 1990.

[11] Collier, F., and Malik, M. R., "Curvature effects on the stability of three-dimensional laminar boundary layers," AGARD CP-438, 1988.

[12] Dando, A. "The Inviscid Compressible Görtler Problem in Three-Dimensional Boundary Layers," Theor. Comp. Fluid Dynamics, Vol. 3, No. 5, pp. 253-367, 1992.

[13] Denier, J. P., Hall, P., and Seddougui, S. O., "On the Receptivity Problem for Görtler Vortices: Vortex Motions Induced by Wall Roughness," ICASE Report 90-31, 1990; also appeared as Phil. Trans. R. Soc. Lond. A., Vol. 335, pp. 51-85, 1991.

[14] Drazin, P. G., and Howard, L. N., "The Instability to Long Waves of Unbounded Parallel Inviscid Flow," J. Fluid Mech. Vol. 14, pp. 257-283, 1962.

[15] Duck, P. W., "The Inviscid Axisymmetric Stability of the Supersonic Flow Along a Circular Cylinder," J. Fluid Mech., Vol. 214, p. 611, 1990.

[16] Duck, P. W., and Hall, P., "On the Interaction of Tollmien-Schlichting Waves in Axisymmetric Supersonic Flows," Q. Jl. Mech. appl. Math, Vol. 42, Pt. 1, pp. 115-130, 1989.

[17] Gaster, M., "On the Effects of Boundary Layer Growth on Flow Stability," J. Fluid Mech., Vol. 66, pp. 465-480, 1974. 
[18] Goldstein, M. E., "Scattering of Acoustic Waves into Tollmien-Schlichting Waves by Small Streamwise Variations in Surface Geometry," J. Fluid Mech.,Vol. 154, pp. 509-529, 1985.

[19] Goldstein, M. E., and Choi, S. W. "Nonlinear Evolution of Interacting Oblique Waves on Two-Dimensional Shear Layers," J. Fluid Mech.,Vol. 207, p. 97, 1989. Corrigendum, J. Fluid Mech.,Vol. 216, p. 659, 1990.

[20] Goldstein, M. E., and Leib, S. J. "Nonlinear Roll-Up of Externally Excited Free Shear Layers," J. Fluid Mech., Vol. 191, p. 481, 1988.

[21] Goldstein, M. E., and Leib, S. J. "Three-Dimensional Boundary Layer Separation Induced by Small-Amplitude Streamwise Vorticity in the Upstream Flow," J. Fluid Mech., Vol. 246, pp. 21-42, 1993a.

[22] Goldstein, M. E., and Leib, S. J. "A Note on the Distortion of a Flat Plate Boundary Layer by Free-Stream Vorticity Normal to the Plate," J. Fluid Mech., Vol. 248, pp. 531-541, 1993b.

[23] Goldstein, M. E., Leib, S. J., and Cowley, S. J., "Distortion of a Flat Plate Boundary Layer by Free-Stream Vorticity Normal to the Plate," J. Fluid Mech., Vol. 237, pp. 231-260, 1992.

[24] Gregory, N., Stuart, J. T., and Walker, W. S. On the Stability of Three Dimensional Boundary Layers with Application to the Flow due to a Rotating Disk," Phil. Trans. R. Soc. Lond. A, Vol. 248, pp. 155-199, 1955.

[25] P. Hall, "Taylor-Görtler vortices in Fully Developed or Boundary Layer Flows: Linear Theory," J. Fluid Mech., Vol. 124, pp. 475-494, 1982.

[26] P. Hall, "The Görtler Vortex Instability Mechanism in Three-Dimensional Boundary Layers," Proc. Roy. Soc. London A., Vol. 399, pp. 135-152, 1985.

[27] Hall, P., "An Asymptotic Investigation of the Stationary Modes of Instability of the Boundary Layer on a Rotating Disk," Proc. Roy. Soc. Lond. A, Vol. 406, p. 93, 1986. 
[28] Hall, P., "Görtler Vortices in Growing Boundary Layers: The Leading Edge Receptivity Problem, Linear Growth and the Nonlinear Breakdown Stage," Mathematika, Vol. 3, pp. 151-189, 1990.

[29] Hall, P., and Smith, F. T., "On Strongly Nonlinear Vortex-Wave Interactions in BoundaryLayer Transition," J. Fluid Mech., Vol. 227, p. 641, 1991.

[30] P. Huerre, and P. A. Monkewitz, "Local and Global Instabilities in Spatially Developing Flows," Ann. Rev. Fluid Mech., Vol. 22, pp. 473-537, 1990.

[31] Malik, M. R., "The Neutral Curve for Stationary Disturbances in Rotating-Disk Flow," J. Fluid Mech., Vol. 164, p. 275, 1986.

[32] Malik, M.R. \& Poll, D.I.A., "Effect of Curvature on Three-Dimensional Boundary Layer Stability," AIAA Journal, Vol. 23, No. 9, pp. 1362-1369, 1985.

[33] Morkovin, M. V., "Critical Evaluation of Transition from Laminar to Turbulent Shear Layers with Emphasis on Hypersonically Traveling Bodies," AFFDL-TR 68-149, 1969.

[34] Papageorgiou, D., and Smith, F. T., "Linear Instability of the Wake behind a Flat Plate," J. Fluid Mech. Vol. 208, pp. 67-89, 1989.

[35] Ruban, A. I., "On the Generation of Tollmien-Schlichting Waves by Sound," Transl. in Fluid Dyn., Vol. 19, pp. 709-16, 1985.

[36] Ryzhov, O. S., and Terent'ev, E. D., "Wave Motions in a Spatial Boundary Layer," J. Appl. Math. Mech., Vol. 55, pp. 789-804, 1991.

[37] F. T. Smith, "On the nonparallel flow stability of the Blasius boundary layer," Proc. Roy. Soc. Lond., Ser. A, Vol. 366, pp. 91-109, 1979.

[38] Stewart, P. A., and Smith, F. T., "Three-Dimensional Instability in Steady and Unsteady Nonparallel Boundary Layers, Including Effects of Tollmien-Schlichting Disturbances and Crossflow," Proc. Roy. Soc. Lond., Ser. A, Vol. 409, pp. 229-248, 1988.

[39] Tam, C. K. W., "The Excitation of Tollmien-Schlichting Waves in Low Subsonic Boundary Layers by Free-Stream Sound Waves," J. Fluid Mech., Vol. 109, pp. 483-501, 1981. 
[40] Timoshin, S. N, "Asymptotic Analysis of a Spatially Unstable Görtler Vortex Spectrum," Mekh. Zidk. Gaza, No. 1, pp. 32-41, 1990.

[41] Zavol'skii, N. A., Reutov, V. P., and Ryboushkina, G. V., "Generation of TollmienSchlichting Waves via Scattering of Acoustic and Vortex Perturbations in Boundary Layer on Wavy Surface," J. Appl. Mech. Techn. Physics, pp. 79-86, 1983.

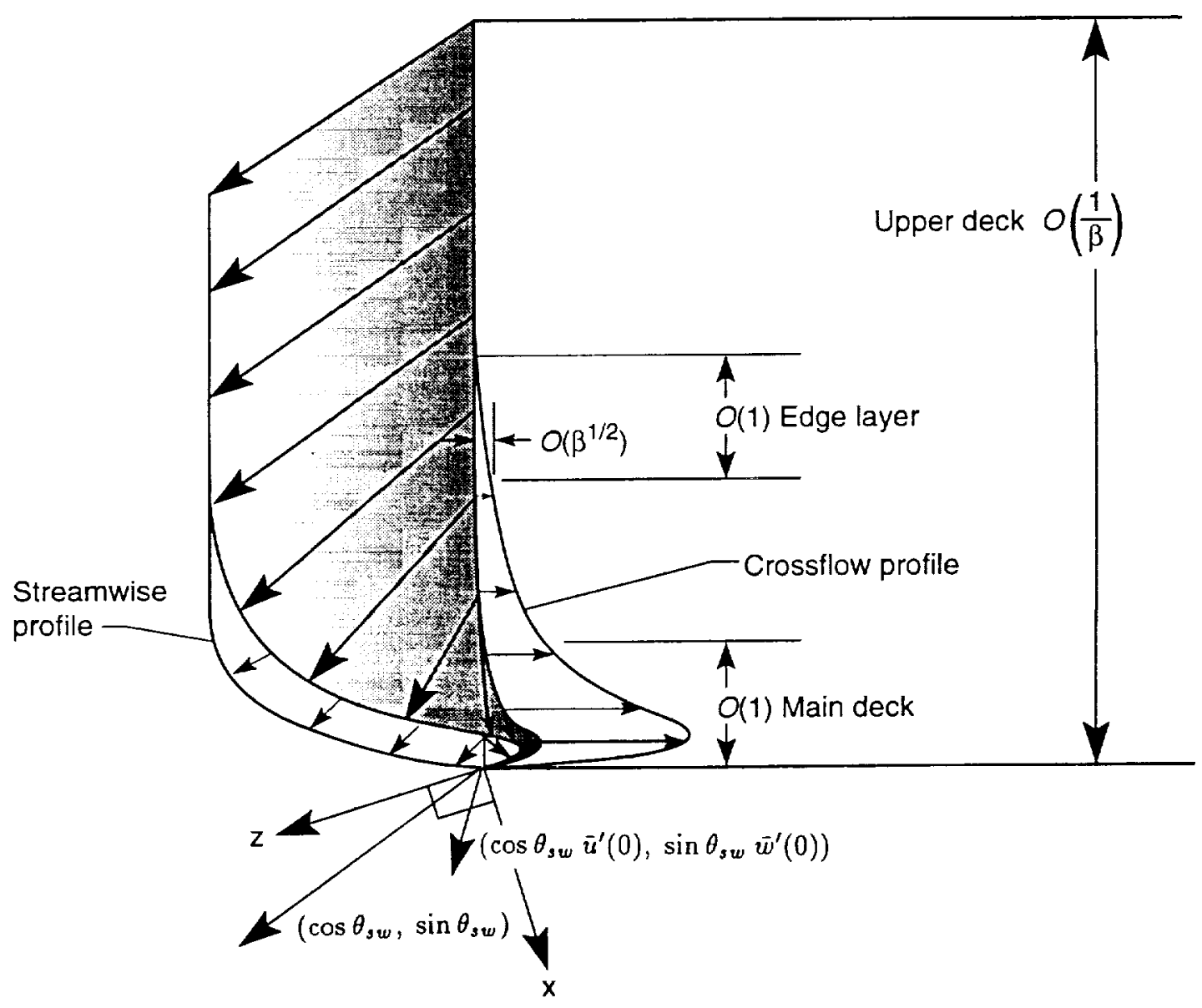

Figure 1. Schematic of asymptotic structure of long-wavelength Rayleigh modes. 


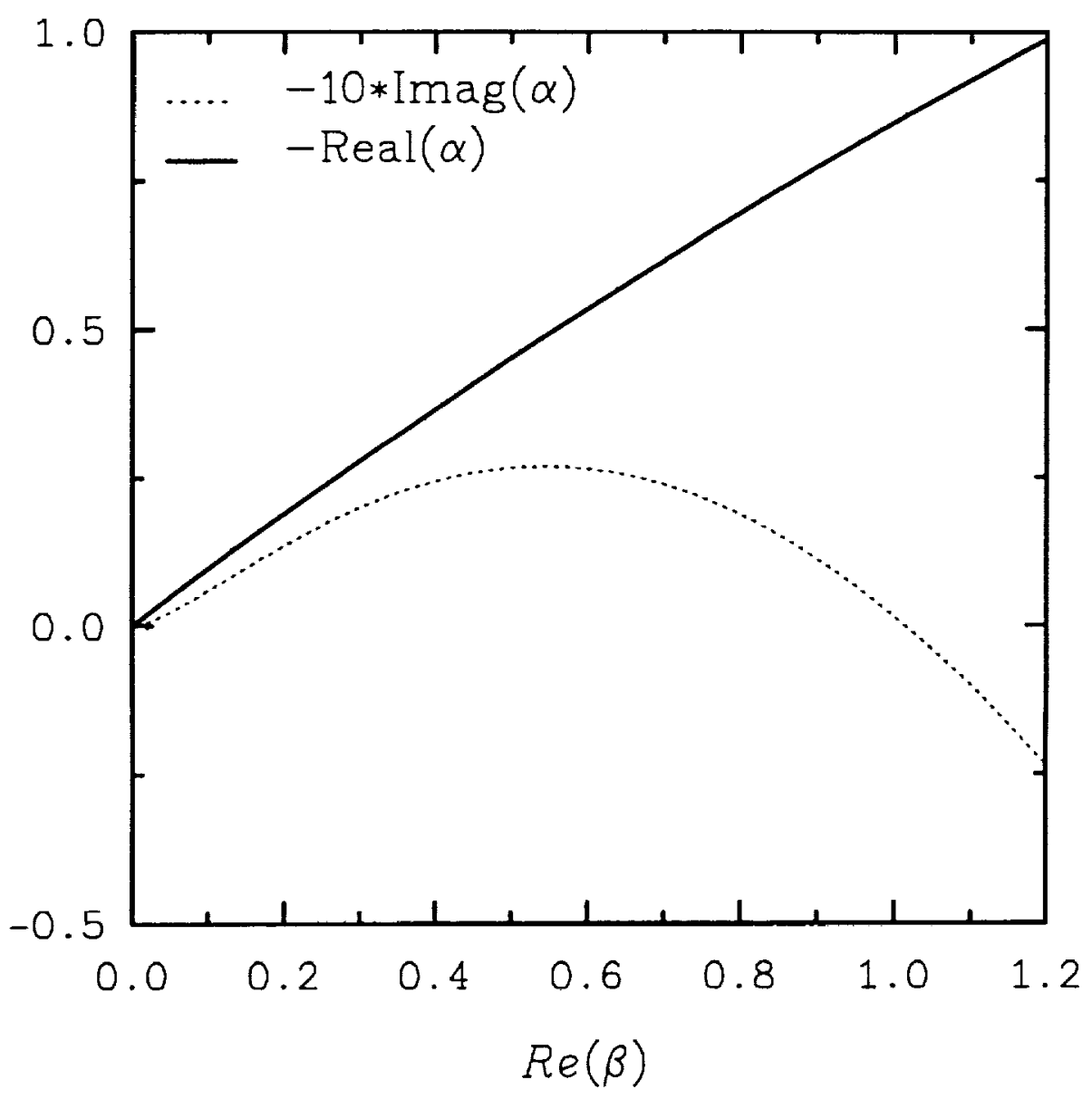

Figure 2(a). Real $(\alpha)$ and $-10 * \operatorname{Imag}(\alpha)$ as functions of wave-number parameter $\beta$ for $\beta_{H}=0.6$ and $\theta_{s w}=45^{\circ}$. 


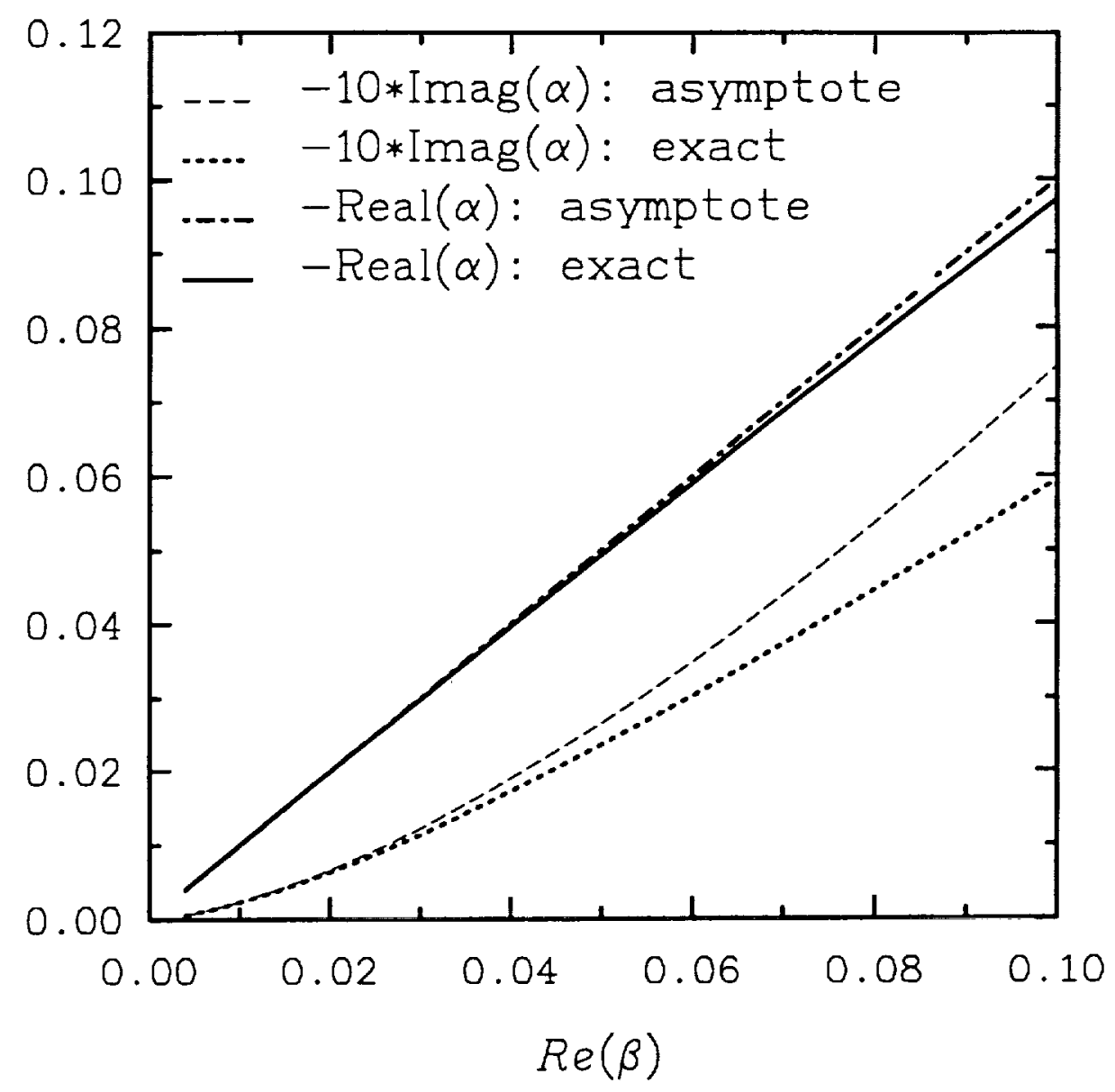

Figure (2b). Long-wavelength asymptotes of curves in figure 2(a) $(-\operatorname{Real}(\alpha)$ and $-10 * \operatorname{Imag}(\alpha)$ as functions of wave-number parameter $\beta$ for $\beta_{H}=0.6$ and $\theta_{s w}=45^{\circ}$. 


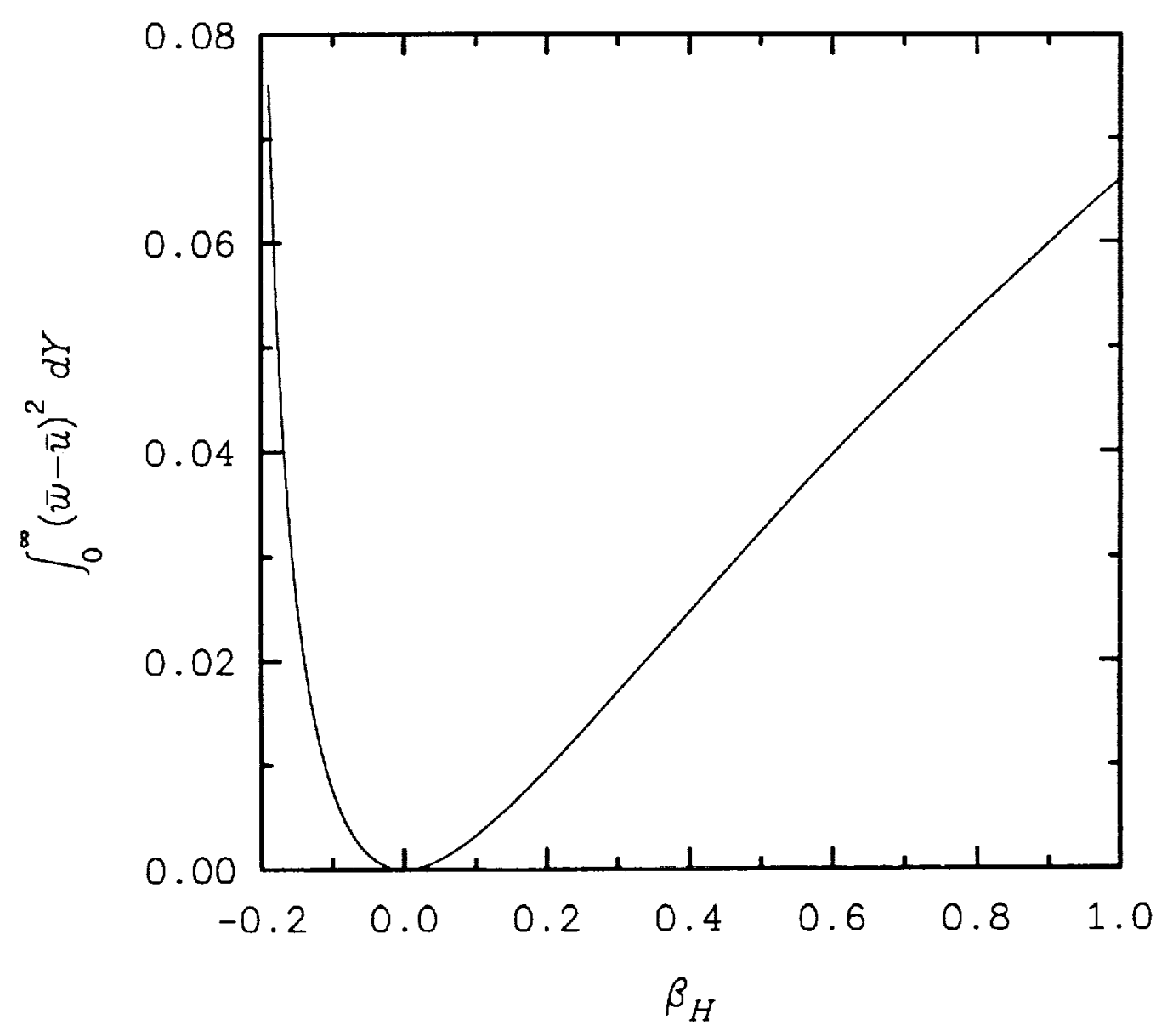

Figure 2(c). Normalized crossflow-momentum integral $\int_{0}^{\infty}(\bar{u}-\bar{u})^{2} d Y$ for FSC profiles as function of Hartree parameter $\beta_{H}$. 


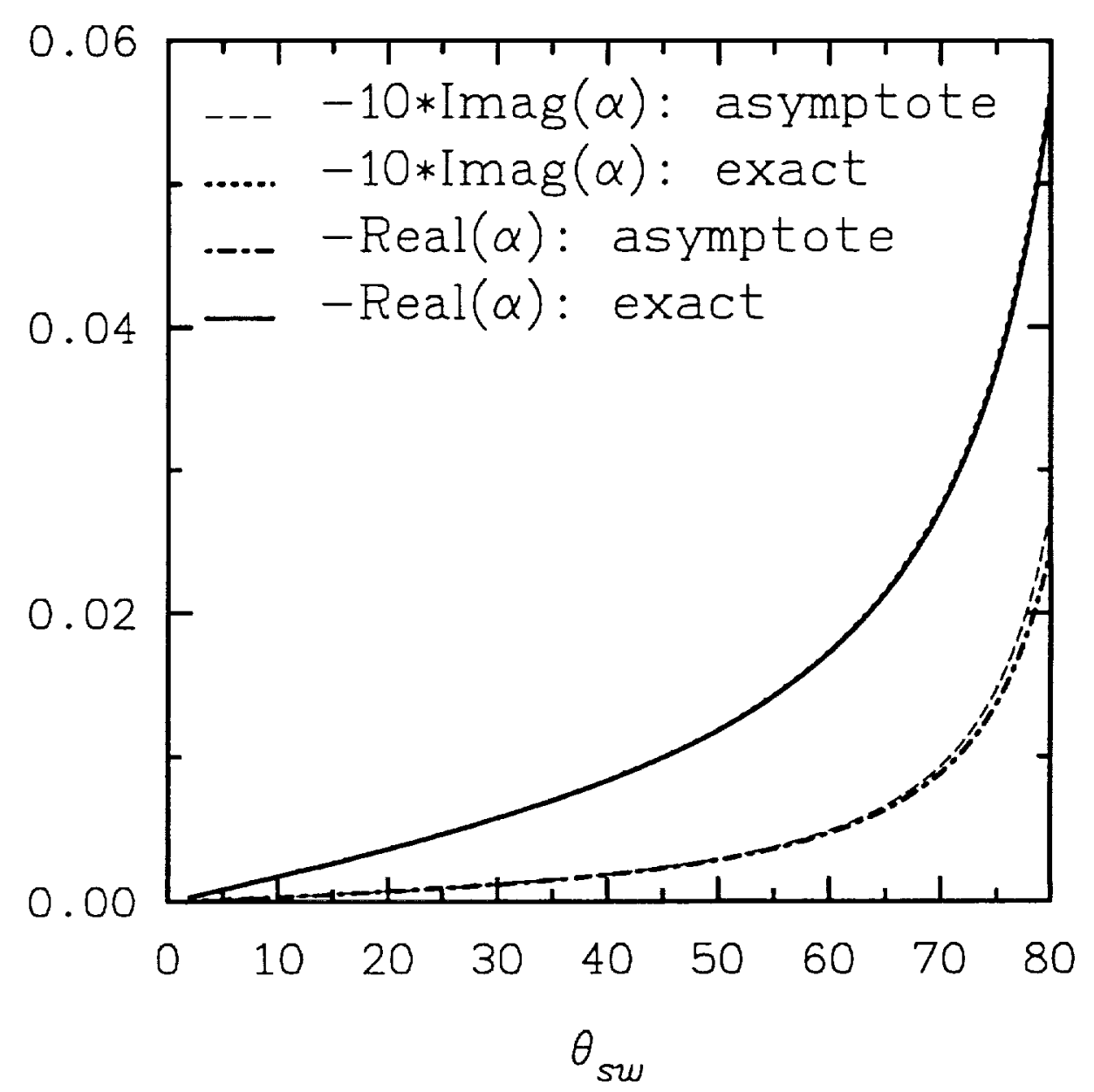

Figure 2(d). $\quad-\operatorname{Real}(\alpha)$ and $-10 * \operatorname{Imag}(\alpha)$ as functions of local sweep angle $\theta_{\text {sw }}$ for $\beta_{H}=0.6$ and $\beta=0.01$. 


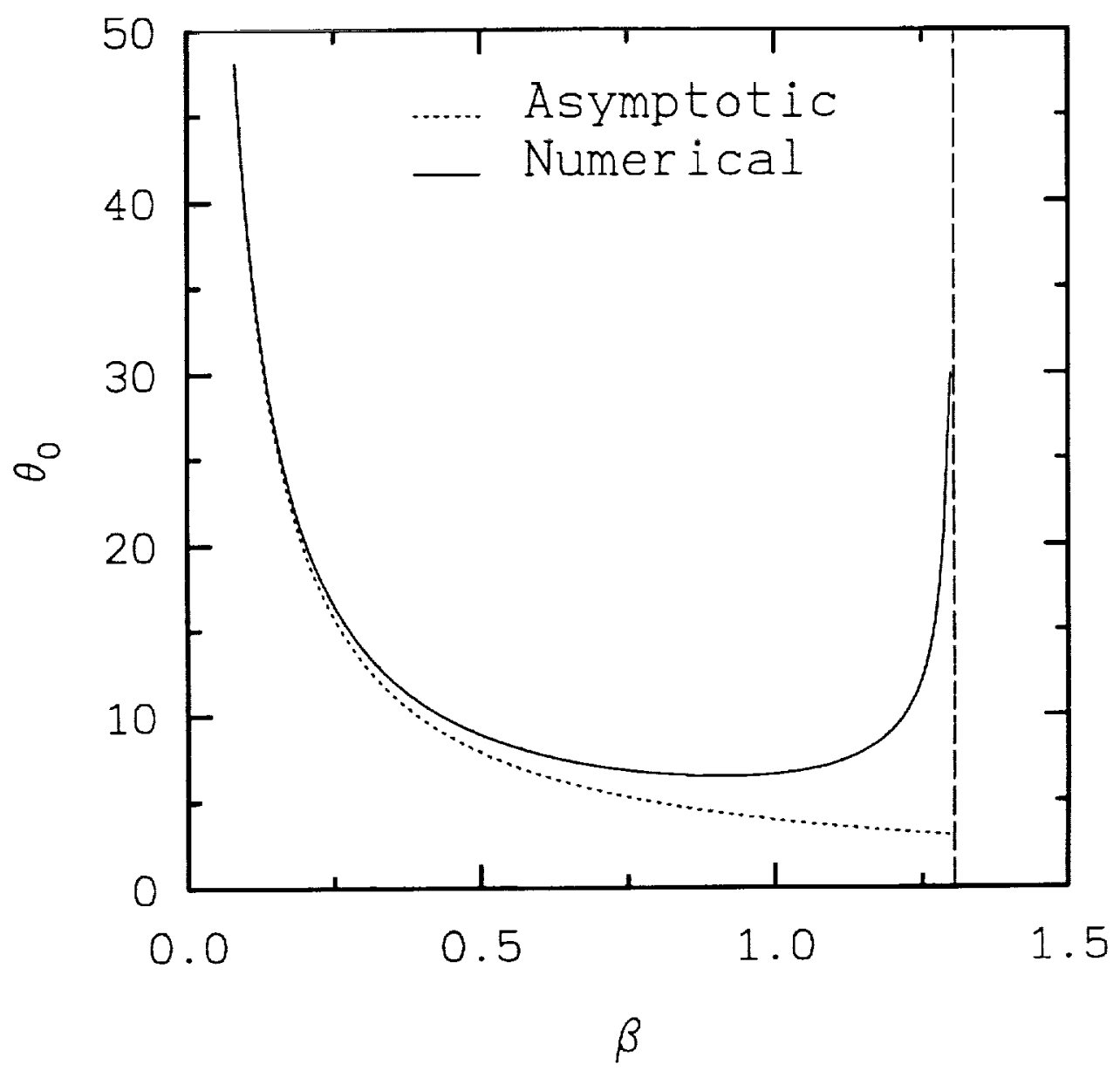

Figure 3. Comparison of long-wavelength asymptote (34) with numerically computed neutral curve (Blackaby and Choudhari, 1993) for stationary vortex disturbances in three-dimensional boundary layer over convex surface. (Upper branch asymptote of Gregory, Stuart, and Walker (1955) is indicated by dashed curve.) 


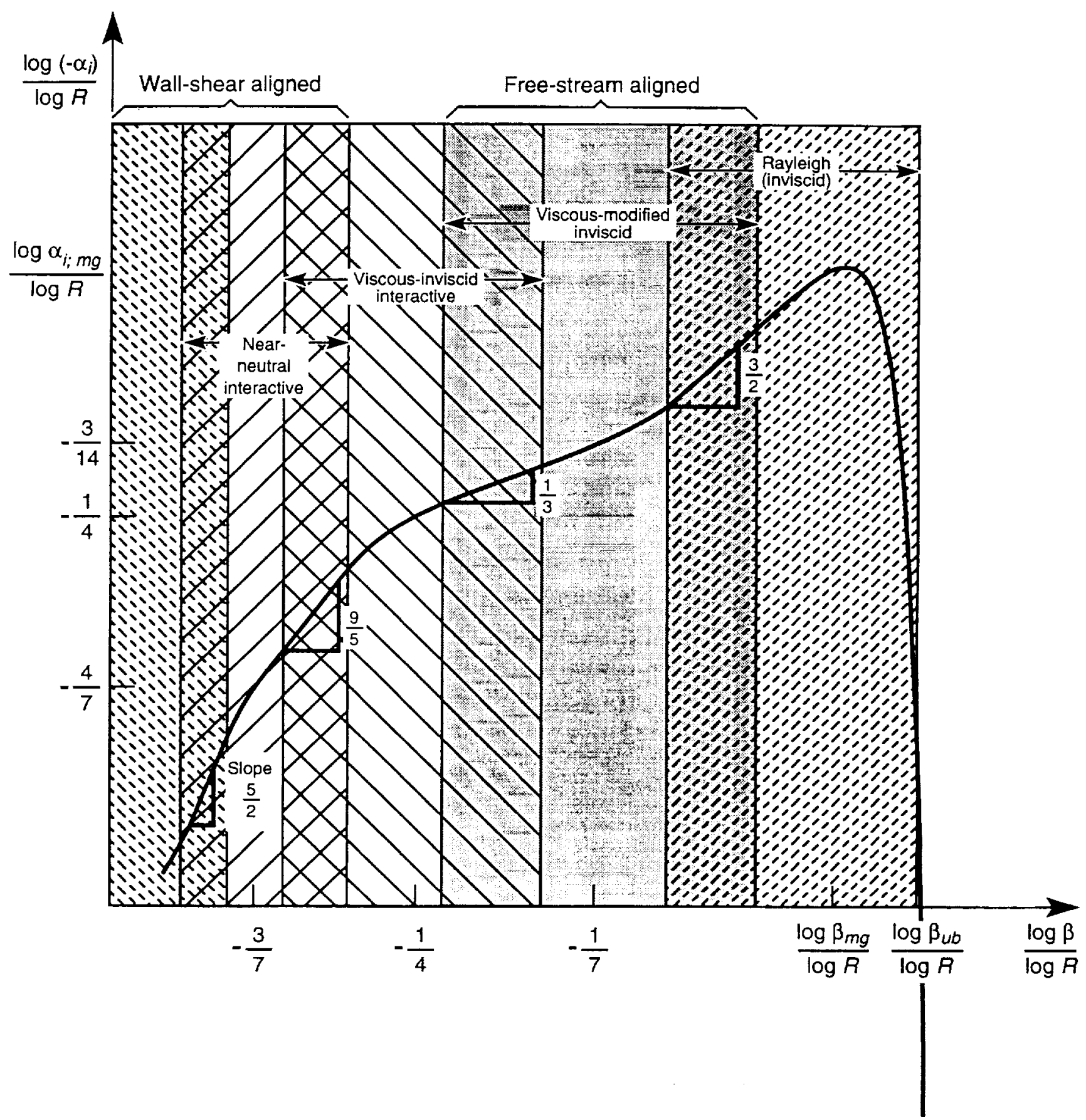

Figure 4. Asymptotic subregimes in stationary crossflow instability in three-dimensional boundary layer over flat surface (sketch not to scale) where $\alpha_{i}$ denotes imaginary part of the eigenvalue parameter $\alpha$, and $\beta_{m g}$ and $\beta_{u b}$ represent wave numbers that correspond to maximum spatial growth rate and upper branch of neutral stability curve, respectively. 


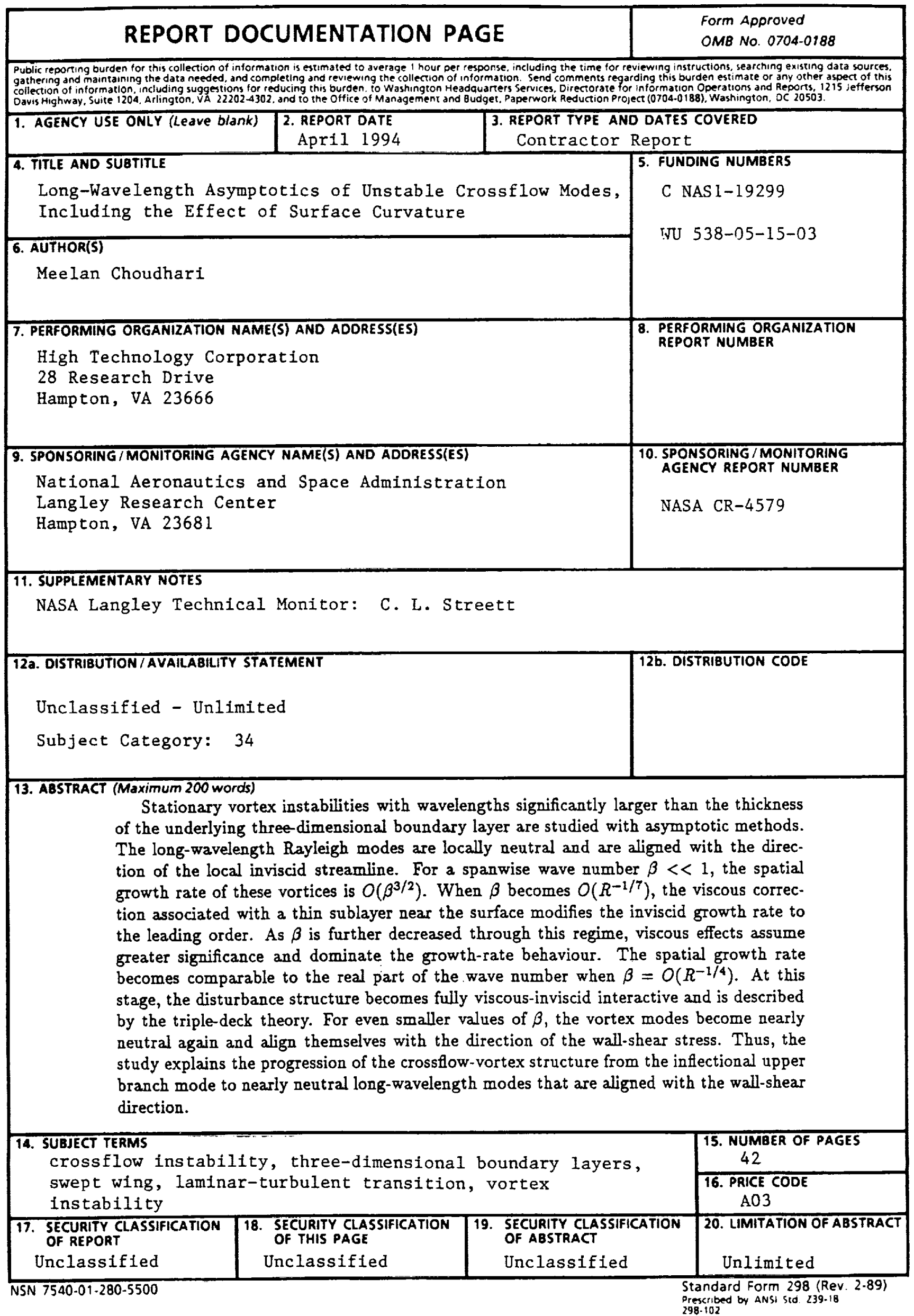

